7-1-2012

\title{
Expertise, Structure, and Reputation of Corporate Boards
}

Doron Levit

University of Pennsylvania

Follow this and additional works at: https://repository.upenn.edu/fnce_papers

Part of the Finance and Financial Management Commons

\section{Recommended Citation}

Levit, D. (2012). Expertise, Structure, and Reputation of Corporate Boards. http://dx.doi.org/10.2139/ ssrn.1866384

This paper is posted at ScholarlyCommons. https://repository.upenn.edu/fnce_papers/13

For more information, please contact repository@pobox.upenn.edu. 


\title{
Expertise, Structure, and Reputation of Corporate Boards
}

\author{
Abstract \\ This paper studies the optimal structure of the board with an emphasis on the expertise of directors. The \\ analysis provides three main results. First, the expertise of a value-maximizing board can harm \\ shareholder value. Second, it is optimal to design a board whose members are biased against the \\ manager, especially when their expertise is high. Third, directors' desire to demonstrate expertise can \\ shift power from the board to the manager on the expense of shareholders. The effect of these reputation \\ concerns is amplified when the communication within the boardroom is transparent.

\section{Keywords} \\ board of directors, communication, information acquisition, expertise, reputation, transparency \\ Disciplines \\ Finance and Financial Management
}




\title{
Expertise, Structure, and Reputation of Corporate
} Boards

\author{
Doron Levit*
}

July 2012

\begin{abstract}
This paper studies the optimal structure of the board with an emphasis on the expertise of directors. The analysis provides three main results. First, the expertise of a value-maximizing board can harm shareholder value. Second, it is optimal to design a board whose members are biased against the manager, especially when their expertise is high. Third, directors' desire to demonstrate expertise can shift power from the board to the manager on the expense of shareholders. The effect of these reputation concerns is amplified when the communication within the boardroom is transparent.
\end{abstract}

Keywords: Board of Directors, Expertise, Communication, Reputation, Information Acquisition, Transparency

JEL Classification Numbers: D74, D82, D83, L14, G34

${ }^{*}$ The author is from the University of Pennsylvania, Wharton School, Finance Department. I am grateful to Philip Bond, Fenghua Song, and Bilge Yilmaz for helpful comments. All errors are mine. Address for correspondence: 3620 Locust Walk, 2433 Steinberg Hall/Dietrich Hall, Philadelphia, PA 19104-6367. Email: dlevit@wharton.upenn.edu 


\section{Introduction}

Shareholders of public companies rely on the board of directors to perform a variety of tasks on their behalf: set strategy, approve major transactions, monitor the senior management, and appoint and replace the CEO. Which type of directors can be expected to serve the best interests of shareholders? Directors with the relevant business expertise are often considered to be natural candidates. Presumably, a mix of industry knowledge, experience, formal education in related disciplines, and social ties, is a recipe for a successful corporate policy. The empirical literature, however, does not fully support this premise. In some cases, it provides the opposite evidence. For example, Guner, Malmendier, and Tate (2008) find that firms with investment bankers on their boards undertake worse acquisitions. ${ }^{1}$ This raises the question whether shareholders should demand high level of expertise from their board members, and if so, under what conditions. More broadly, understanding the optimal structure of the board has become particularity important in light of recent trends of corporate governance which have given shareholders more power and influence on the composition of corporate boards. ${ }^{2}$

This paper studies three topics that are related to the expertise of directors: the optimal level of expertise on the board, the interaction between expertise and the affiliation of directors, and the effect of directors' reputation concerns for being experts on the value of the firm. By focusing on the expertise of directors this paper offers a new perspective on the optimal structure of corporate boards.

In order to study this topic I develop a model of board's expertise. In the model, the board

\footnotetext{
${ }^{1}$ In addition, Defond, Hann, and $\mathrm{Hu}(2005)$ document a positive stock market reaction to the appointment of directors with accounting knowledge to the audit committee, but not to the appointment of other financial experts. Fich (2005) finds that the cumulative abnormal return in response to the addition of a director who is CEO of another firm is significantly greater the higher the industry-adjusted ROA of his firm. However, the effect is insignificant and sometimes even negative, if the new director holds a MBA or is an academic.

${ }^{2}$ For example, the increased role played by proxy advisory firms in directors elections (Alexander et al. (2008)), the debate on whether shareholders should be granted with an easier proxy access (for more details see, for example, Fisch (2011)), the shift from plurality to majority voting system in directors elections (Kahan and Rock (2010)), and the Proxy Disclosure Enhancements regulation which was adopted by the SEC in 2009 and requires companies to provide new disclosure about the background and qualifications of directors and nominees.
} 
decides whether to invest in a project based on the expertise of its directors and the recommendation given by the manager of the company. The expertise of the board is measured by the quality of its private information about the profitability of the firm's investment opportunity. The manager, by the nature of his job, can produce valuable and private information which is unavailable to the board. The production and communication of this information do not impose direct costs on the manager. However, because of conflicting preferences, the manager may still choose not to produce or share information. Board members (at least partly) observe the manager's effort to produce information, but unless the manager discloses his finding, they cannot observe the content of this information. Without this information, directors must rely on their own judgment when making decisions, circumstances in which their expertise is relatively more important. Thus, the model reflects the view that the expertise of directors matters the most when the manager does not cooperate with the board.

All else equal, shareholders are better off with a higher level of board's expertise. However, the first result of the paper demonstrates that when the incentives of the manager to produce and share information with the board are considered, the expertise of a value-maximizing board can harm shareholder value.

In order to understand this result, note that expertise of the board has two countervailing effects. The obvious effect of expertise is positive, it reduces the likelihood the board chooses a sub-optimal investment policy. The negative effect is more subtle. I show that even though the production of information is costless, the board's expertise can discourage the manager's incentives to produce and share information. Basically, since managerial effort is observable by the board, learning increases the likelihood the board ignores the recommendation of the manager when the latter conceals information. Expertise interacts with this prejudice effect and may increase it. In order to minimize this effect, the manager invests less effort in learning. To the extent that the manager's information is relatively more important than the expertise of the board, the negative effect dominates and a higher level of board's expertise is associated with 
a lower shareholder value. This result challenges the view that more expertise on corporate boards necessarily improves firm's policy, and it is consistent with the ambiguous empirical evidence on the value of directors' expertise.

The second result of this paper provides conditions under which the optimal structure of the board consists of directors whose objective is misaligned with value-maximization. It demonstrates that as long as the manager has sufficient access to information, it is never optimal to structure a board whose members are affiliated with the manager. Instead, if directors have high expertise, the optimal affiliation of the board is against the agenda of the manager. Thus, by contrast to the existing literature on corporate boards, this result implies that shareholders can benefit from supporting nominees for the board who are inherently biased against the incumbent management and would frequently challenge its view. ${ }^{3}$

Intuitively, when the bias of the board is directed against the manager, the board would follow the recommendation of the manager only if the latter provides solid evidence to backup his recommendation. Therefore, the manager has more incentives to produce and share information with a biased board. When choosing the structure of the board, shareholders trade off between informed and distorted board decision making. It is optimal to appoint directors who are biased against the manager when the latter has sufficient access to information but his incentives to produce and share it with the board are otherwise weak.

The third result of the paper demonstrates that the desire of directors to demonstrate expertise can shift power from the board to the manager and result with a lower shareholder value. ${ }^{4}$ In particular, concerns for reputation distort the board's behavior from maximizing the value of shareholders and the board under-reacts to public information. This distortion is exploited

\footnotetext{
${ }^{3}$ For example, Adams and Ferreira (2007) and Almazan and Suarez (2003) argue that boards which are affiliated with the incumbent management are optimal. One exception is Baranchuk and Dybvig (2009) who use a cooperative solution concept to study directors' collective decision making. They show that sometimes it is better to add a new informed director to the board who has conflicted preferences with insiders than adding an uninformed and independent director.

${ }^{4}$ While the first result of the paper shows that expertise can adversely affect the value of the firm, directors still have incentives to demonstrate expertise when the demand for expertise varies across firms and directors can simultaneously serve on more than one board.
} 
by the manager, and hence, the direction and the magnitude of the board's bias relative to the manager's agenda are determined in equilibrium. In this sense, the "friendliness" of the board is endogenous since it depends on the decision of the manager to produce and share information with the board and the perception of the board's quality by its shareholders. Interestingly, the effect of directors' reputation concerns on managerial cooperation and the value of shareholders is amplified by the transparency of the communication within the boardroom. This is because transparency enables shareholders to tell apart between decisions the board makes based on its expertise and decisions which are taken based on the manager's recommendation.

Overall, the composition of the results in this paper sheds light on new considerations that ought to be taken into account by shareholders and policy makers when designing the structure of corporate boards. The analysis suggests that demanding a minimum level of expertise from independent directors (to be eligible to serve on the board of a public company) can harm shareholders even when the conflict of interests with their manager is significant. In those circumstances, it may be useful to encourage the appointment of directors whose agenda is not necessarily consistent with maximizing shareholders' value, but it contrasts the incumbent management. Labor unions and hedge funds are examples for potential dissenting voices on corporate boards. Moreover, putting directors under pressure, a reflection of recent trends in the corporate governance of public U.S. companies, can enhance the incentives of directors to manage their reputation and demonstrate expertise. Consequently, instead of more discipline, managers of public companies might gain more power. This suggests that the amount of control that shareholders have on the identity of elected directors should be treated with caution. Finally, promoting a policy which would require more transparency of boardroom minutes can in fact reduce the amount of information that is available to shareholders and the board itself, even if information is not proprietary. Thus, transparency can adversely affect the value of the firm.

The paper proceeds as follows. The remainder of this section discusses the relationship 
to the existing literature. Section I presents the baseline model in which the board is valuemaximizing and has no reputation concerns. Section II analyzes optimal board affiliation and its interaction with directors' expertise. Section III introduces board's concerns for reputation and studies their effect on the interaction in the boardroom and shareholder value. Section IV provides concluding remarks and discusses the policy implications and the empirical predictions of the analysis in this paper. All omitted proofs and supplemental results are collected in the Appendix.

\section{Relation to the Literature}

The present paper is related to the agency literature on informed principal. A key observation of this literature is that information flows alleviate an adverse selection problem (it is easier to detect agent's type) but also create a moral hazard problem (the agent exerts less costly effort). ${ }^{5}$ In the context of corporate boards, Hermalin and Weisbach (2012) argue that managers will need to be compensated for the harm caused them by the expert board, and that the increase in compensation is the cost of having an expert board. Levit (2011) demonstrates that recommendations given to target shareholders by their informed board can deter valueincreasing bidders. Thus, in the context of takeovers, uninformed target boards may create more value to their shareholders than an informed board. Different from these studies, the task of the agent (the company's manager) in the present model is to collect information (at no direct cost) and then communicate it to the partly informed principal (the board). Therefore, the channel through which expertise can be harmful is fundamentally different in the present paper.

The current study is also related to Che and Kartik (2009) and Kamenica and Gentzkow (2011). Che and Kartik (2009) study a model in which the agent produces information (at

\footnotetext{
${ }^{5}$ For example, see Burkart, Gromb, and Panunzi (1997), Cremer (1995), Dewatripont, Jewitt and Tirole (1999), Holmstrom (1999), Rajan (1992), and Von Thadden (1995). Different from these studies, Prat (2005) shows that the transparency of actions taken by an agent with reputation concerns for his expertise can create incentives for the agent to disregard useful private information.
} 
a cost) and then voluntarily disclose it to an uninformed decision maker. Their key result shows that difference of opinions ("agreeing to disagree") can help to alleviate the hold-up problem of a costly information acquisition. By contrast, the present study abstracts from these hold-up motives and focuses on the interaction between the precision of the decision maker's private information and the incentives of the agent to collect and disclose additional information. Kamenica and Gentzkow (2011) show that the agent can benefit from disclosure of information if the principal does not take the agent's preferred action by default. Key to their results is the agent's inability to conceal information once the signal realization is known. The present paper extends their framework by showing that the quality of the principal's private information can deprive the agent's incentives to collect information even when the latter can conceal it from the principal. ${ }^{6}$

Adams and Ferreira (2007) study the dual role of the board as advisor and monitor of management. They argue that in order to obtain better information from managers, the board may want to commit not to use this information in evaluating managerial performance. In this sense, they argue, friendly boards are optimal. Chakraborty and Yilmaz (2011) and Harris and Raviv (2005, 2008, 2010) employ two-sided private information in the Crawford and Sobel (1982) model of strategic information transmission, and analyze optimal delegation between the board (or shareholders) and the manager. Unlike the present paper, in these studies information is non-verifiable, and hence, misalignment of preferences always reduces the amount of information that is disclosed in equilibrium. Moreover, in the above studies the board's information is incremental to the manager' information, while in the present paper the board's expertise is an imperfect substitute.

In the context of reputation, Hirshleifer and Thakor (1998) show that if the board is sufficiently concerned about its own job security, the threat of a hostile takeover can pressure the

\footnotetext{
${ }^{6}$ In this respect, the present model builds on Dye's (1990) model of voluntary disclosure which proposes that uncertainty of whether the sender is informed allows senders with bad news to claim they have no new information, rather than disclose it. The present paper endogenizes the information endowment of the sender.
} 
board to fire the manager even when it is suboptimal. Ruiz-Verdu and Singh (2011) show that in order to be perceived as independent, boards lower managers' pay, but may also pay managers in hidden and inefficient ways. Different from these studies, the present paper highlights that the board's concerns for reputation can be exploited by the opportunistic manager and thereby lead to inefficient decisions. Song and Thakor (2006) study a model of information transmission in which the tension between the manager and the board is the outcome of the their conflicting career concerns. Unlike the present paper, in their model the manager cannot collect information and the board can add value since it obtains information that is unavailable to the manager.

\section{A Model of Board's Expertise}

\section{A Setup}

Consider a firm whose value to shareholders depends on its business strategy and the fundamentals. Let $a \in\{0,1\}$ be the strategy, $\omega \in\{0,1\}$ the true state of the world, and $p \equiv \operatorname{Pr}[\omega=1] \in$ $(0,1)$ the common prior on $\omega$. The value of the firm is given by $v(a, \omega)=1_{\{a=\omega\}}$. Thus, when $\omega=1$ the optimal strategy is $a=1$ and when $\omega=0$ the optimal strategy is $a=0$. For example, the relative value of growing the firm through acquisitions (rather than expanding it internally) is a function of its organizational culture and the supply of potential targets. While strategy $a$ can relate to the firm's investment policy, capital structure, or corporate governance, in what follows I refer to decision $a$ as investment and say that the project is approved when $a=1$ and the project is rejected otherwise.

The firm is run by a manager whose preferences are given by $u_{M}(a, \omega)=1_{\{a=1\}}$. That is, the manager derives direct utility from investing in the project regardless of how much value it generates to shareholders. For example, if $a=1$ represents growth through acquisitions then an

empire-building manager benefits from acquiring other firms even if value is destroyed. Since 
the manager is self-interested, the decision making authority over $a$ is allocated to the board of directors. ${ }^{7}$ The board is monolithic and the manager does not have a seat on the board. The board's objective is to maximize value for shareholders and its preferences are given by $u_{B}(a, \omega)=v(a, \omega)$. All preferences are common knowledge.

Shareholders are uninformed about the fundamentals of the firm $\omega$, but their board has expertise which reflects directors' experience (e.g., former executives and directors in other companies) and profession (e.g., lawyers, accountants, bankers, academics, and former politicians). High expertise is associated with a higher likelihood the board obtains private information about $\omega$. Specifically, with probability $\lambda \in[0,1]$ the board observes an informative signal $s$ on $\omega$ and with probability $1-\lambda$ the board remains uninformed. The likelihood the board is informed is independent of $\omega$, and whether the board is informed and the content of signal $s$ are the board's private information. The board does not micro-manage the firm, and hence, has a limited access to information. I therefore assume that the board's private signal is imperfect. Specifically, let $f_{\omega}$ and $F_{\omega}$ be the density and the cumulative functions, respectively, for the probability of receiving signal $s \in[\underline{s}, \bar{s}]$ if the true state of the world is $\omega$. The functions $f_{\omega}(\cdot)$ are continuous and the likelihood function $l(s) \equiv \frac{f_{1}(s)}{f_{0}(s)}$ is strictly increasing. Moreover, $\lim _{s \rightarrow \bar{s}} l(s)=\infty$ and $\lim _{s \rightarrow \underline{s}} l(s)=0$. Thus, without additional information, the board's decision whether to approve the project depends on its private information. ${ }^{8}$

Unlike the board, the manager has free access to information and he can learn about $\omega$ at no personal cost. ${ }^{9}$ For example, the manager can invest in research and development, question his subordinates, solicit consultants' opinions, or engage in competitive intelligence, in order to "test" and explore the project's profitability. These investments in information generate signals about the quality of the project. The precision and verifiability of these signals are increasing

\footnotetext{
${ }^{7}$ While studying optimal delegation is not the focus of the present paper, it can be shown that it is never optimal (from shareholders' point of view) to delegate the decision making to the manager.

${ }^{8}$ The assumption on the limit properties of $l(\cdot)$ is not necessary for the main results but it simplifies the exposition. The crucial assumption is the existence of $\hat{s} \in(\underline{s}, \bar{s})$ such that $l(\hat{s})=1$.

${ }^{9}$ The main results continue to hold even if learning is costly to the manager. However, by abstracting from hold-up problems a la Cremer (1995), the analysis reveals new channel through which expertise can be harmful.
} 
in $e \in[0, \bar{e}]$, the resources expended on learning. Parameter $\bar{e} \in(0,1]$ reflects the access the manager has to information. By learning, the manager obtains a non-verifiable signal $\xi_{e}$ on $\omega$ where $g_{e}(\cdot \mid \omega)$ is its conditional density function. In addition, with probability $e$ the manager obtains a verifiable signal $\tau_{e}$ on $\omega$ and with probability $1-e$ the manager does not obtain a verifiable signal. Signal $\tau_{e}$ is a sufficient statistic for $\omega$ with respect to the board's signal $s$. That is, $\operatorname{Pr}\left[\omega=1 \mid \tau_{e}=\tau, s\right]=\operatorname{Pr}\left[\omega=1 \mid \tau_{e}=\tau\right]$ for all $\tau$ and $s$. This reflects the underlining assumption that the manager can obtain "hard" information which is relatively more important than the board's expertise. ${ }^{10}$ For simplicity, I assume $\tau_{e} \equiv \omega$. Let $I_{e}$ be the information set of the manager, then $I_{e}=\left\{\xi_{e}, \omega\right\}$ with probability $e$ and $I_{e}=\left\{\xi_{e}\right\}$ otherwise. $^{11}$ The content of $I_{e}$ is the manager's private information.

The manager cannot dictate the strategy of the firm, but he can use his informational advantage in order to influence the board's decision. I assume the manager sends the board a message $m=\left(m_{1}, m_{2}\right)$ before the board decides on $a$. The content of message $m_{1}$ is verifiable. Verifiable information cannot be manipulated and any attempt to present a falsified presentation fails. Thus, if the manager observes $\omega$, he can either set $m_{1}=\omega$ or conceal this information by sending the board message $m_{1}=\phi$. If the manager does not observe $\omega$, his only option is sending $m_{1}=\phi$. By contrast, the content of message $m_{2}$ is non-verifiable. Hence, $m_{2}$ can be manipulated and take any value in $[0,1]$ regardless of $I_{e} \cdot{ }^{12}$ Let $\sigma_{e}\left(m_{1}, m_{2} \mid I_{e}\right)$ be the probability the manager sends verifiable message $m_{1}$ and non-verifiable message $m_{2}$ conditional on learning intensity $e$ and the manager's private information $I_{e}$. Note that by assumption, if $\omega \notin I_{e}$ then $\sigma_{e}\left(1, m_{2} \mid I_{e}\right)=\sigma_{e}\left(0, m_{2} \mid I_{e}\right)=0$ and if $\omega \in I_{e}$ then $\sigma_{e}\left(1-\omega, m_{2} \mid I_{e}\right)=0$. I assume the message

\footnotetext{
${ }^{10}$ Note that if $\bar{e}<1$ the board's expertise complements the manager's access to information. The results of the paper continue to hold even if the board has information which is never available to the manager, as long as the complementary of this information is not significant.

${ }^{11}$ Formally, $I_{e}=\left\{\xi_{e}, \zeta_{e}\right\}$ where $\zeta_{e} \in\{0,1, \phi\}, \zeta_{e}=\omega$ with probability $e$, and $\zeta_{e}=\phi$ otherwise. The manager cannot "prove" he does not have verifiable information on $\omega$. Thus, while $\zeta_{e}=\omega$ is verifiable, $\zeta_{e}=\phi$ is not.

${ }^{12}$ To fix ideas, if the agenda is the acquisition of a competitor, the manager can provide the board with "hard" information such as valuation models about expected synergies, financing commitments, lawyers' opinion about the regulatory risk, and so on. At the same time, the manager can provide "soft" information about the friendliness of the target's management to the deal and the moral of employees.
} 
$m=\left(m_{1}, m_{2}\right)$ has no direct effect on the manager's utility. Thus, there are no costs or benefits from disclosure or manipulation per-se.

The board relies on several sources of information when making decisions: public information, its own expertise, and the manager's report $m$. I denote by $a_{e, m}(s)$ the probability the board decides on $a=1$ given this information. ${ }^{13}$ An important assumption of the model is that the manager's learning intensity $e$ is observable by the board but cannot be explicitly contracted. In the context of board-manager interaction this assumption seems reasonable. For example, learning about the benefit from an acquisition requires the manager to spend time meeting with the target's directors and senior management, consulting investment bankers and lawyers, and questioning the company's employees, costumers, and suppliers, about the feasibility of a successful integration. These meetings (their existence but not their content) are likely to be at least partly observed and noticed within the firm and by the board. At the same time, projects (or acquisitions) are different along many dimensions. Effective learning requires the manager to approach a different set of people, ask a different set of questions, and integrate the answers using different methodologies. All of which are hard to specify ex-ante when writing a contract. ${ }^{14}$

\begin{tabular}{|c|c|c|c|}
\hline & & & 1 \\
\hline $\begin{array}{l}\text { Time 0: } \\
\text { The manager decides on } \\
\text { his learning intensity } \boldsymbol{e}\end{array}$ & $\begin{array}{l}\text { Time 1: } \\
\text { The manager obtains signals } \\
\text { on } \boldsymbol{\omega} \text { and sends the board } \\
\text { message } \boldsymbol{m}\end{array}$ & $\begin{array}{l}\text { Time 2: } \\
\text { Based on message } \boldsymbol{m} \text {, signal } \\
\boldsymbol{s} \text {, and its expertise, the board } \\
\text { decides on } \boldsymbol{a} \text {, whether to } \\
\text { accept the project }\end{array}$ & $\begin{array}{l}\text { Time 3: } \\
\text { State } \boldsymbol{\omega} \text { and the value of the } \\
\text { firm } v(a, \omega) \text { are realized. }\end{array}$ \\
\hline
\end{tabular}

Figure 1 - Timeline

\footnotetext{
${ }^{13}$ Notation is simplified by assuming that the uninformed board receives signal $\hat{s}$ with probability one.

${ }^{14}$ I follow the common approach of incomplete contracting by Grossman and Hart (1986) by assuming the board cannot use monetary transfers that are perfectly contingent on the information or effort provided by the manager. In this respect, the main results of this paper only require that the board observes $e$ with a non-zero probability and that high levels of effort cannot be contracted. Since there are no direct costs for effort, if $e$ is completely unobserved by the board then in any equilibrium the manager chooses $e=\bar{e}$.
} 
Overall, the sequence of events unfolds as follows. At the outset the manager decides on his learning intensity $e$. The manager cannot commit to any disclosure strategy. Thus, given his decision $e$ and his private information $I_{e}$, the manager sends the board a private message $m=\left(m_{1}, m_{2}\right)$. Based on all sources of information, the board decides on the probability $a_{e, m}(s)$ that the project is approved. Last, the state of the world $\omega$ and the outcome of the project are realized.

Before we turn into the analysis it is important to note that the board cannot commit to decision rules which are ex-post inefficient conditional on the information it obtains. For example, a commitment to approving the project if and only if the manager discloses $\omega=1$ is ruled out. If allowed, this commitment can enhance the incentives of the manager to collect and share information. Yet, even this strong commitment will not achieve the first best as long as $\bar{e}<1$. In this respect, lack of expertise, affiliation, and reputation concerns, are mechanisms that under certain conditions implement a similar commitment power.

\section{B Analysis}

The model is solved backward. For each level of learning intensity $e$ there exists an induced game with incomplete and asymmetric information which is denoted by $\mathbb{G}(e)$. I start by solving for the Perfect Bayesian Equilibria of $\mathbb{G}(e)$.

Consider the board's decision whether to approve the project. The board is maximizing the value of shareholders. Thus, the board approves the project if and only if given its information

the probability that $\omega=1$ is greater than $\frac{1}{2}$. This decision rule is ex-post efficient from shareholders' point of view. Recall the board's information is nested into the manager's information. Thus, if the manager discloses verifiable information about $\omega$ (and hence $m_{1} \in\{0,1\}$ ), the board always finds it optimal to defer to the manager's view and choose $a=m_{1}$. If the manager does not disclose verifiable information about $\omega$ then the board forms its beliefs about $\omega$ given its private signal $s$, the manager's non-verifiable message $m_{2}$, the knowledge of $e$, and the expec- 
tations that the manager would follow disclosure strategy $\sigma_{e}$. Let $q \equiv \operatorname{Pr}\left[\omega=1 \mid e, m_{1}=\phi, m_{2}\right]$ be the posterior probability that $\omega=1$ conditional on non-disclosure of verifiable information by the manager, but unconditional on signal $s$. Applying Bayes' rules, for any $s \in[\underline{s}, \bar{s}]$ the board's posterior beliefs are given by $\frac{l(s)}{l(s)+(1-q) / q}$. The board approves the project if and only if $s \geq s^{*}(q)$ where $s^{*}(q) \equiv l^{-1}((1-q) / q)$. The pair $(s, q)$ is a sufficient statistic for the information that is available to the board conditional on non-disclosure of verifiable information by the manager. The following lemma summarizes the above discussion and characterizes equilibria of the sub-game that is induced by the manager's learning intensity $e$.

Lemma 1 In any equilibrium of the induced game $\mathbb{G}(e)$ :

(i) The manager discloses verifiable information if $\omega=1$ and withholds it if $\omega=0$.

(ii) Non-verifiable information is revealed only if the manager discloses verifiable information.

(iii) If $m_{1} \neq \phi$ then $a_{e, m}^{*}(s)=m_{1}$ and otherwise $a_{e, m}^{*}(s)=1_{\left\{s \geq s^{*}(q(e))\right\}}$ where $q(e) \equiv \frac{p-e p}{1-e p} .15$

Lemma 1 has several implications for the manager's disclosure policy. First, in any equilibrium the manager has correct expectations regrading the board's reaction to his disclosure decision. In particular, the manager realizes that if he discloses verifiable information about $\omega$ the board will choose $a=\omega$ with probability one. Thus, if the manager obtains hard evidence on $\omega$, he always has strict incentives to disclose $\omega=1$ and weak incentives to conceal $\omega=0$.

Second, Lemma 1 implies that there is no equilibrium in which the manager is able to influence the board's decision by communicating non-verifiable information about $\omega$. To see why, recall the manager's objective is to maximize the probability the project is approved by the board. Thus, if the manager discloses verifiable information about $\omega$, any additional information is redundant and cannot change the board's decision. If instead the manager

\footnotetext{
${ }^{15}$ Unless $m_{1}=\phi$ and $s=s^{*}(q)$, the board's optimal decision is unique. It is implicitly assumed that the board plays pure strategies.
} 
does not disclose verifiable information about $\omega$, the manager has strict incentives to send the non-verifiable message that leads to the highest probability of approval. This implies that in equilibrium any non-verifiable message must lead to the same action and information about $\xi_{e}$ cannot be revealed. ${ }^{16}$

Third, if the manager does not disclose verifiable information, part ( $i i)$ of Lemma 1 suggests that the board's decision depends solely on its expertise and its inference from the manager's decision not to disclose information. To the extent that $e<1$, the manager does not disclose verifiable information about $\omega$ either because he does not obtain such information or because he strategically conceals this information. The board accounts for both possibilities and adjusts its beliefs accordingly. In particular, since the manager is biased toward the approval of the project, non-disclosure always conveys information about its non-profitability and $q(e) \leq p$. Moreover, $q(e)$ is decreasing in $e$ which implies that the board put relatively more weight on the event that the manager strategically conceals information about $\omega=0$ the higher is the managerial learning intensity.

Given the characterization of the equilibrium of the induced game $\mathbb{G}(e)$, I turn into studying the manager's learning intensity decision. Since information about $\xi_{e}$ cannot be credibly communicated, hereafter I refer to learning only with respect to verifiable information about $\omega$. Let $\alpha(q, x)$ be the ex-ante probability the informed board approves the project if an outsider believes that $\operatorname{Pr}[\omega=1]=x$ and prior to observing signal $s$ the board believes that $\operatorname{Pr}[\omega=1]=q$. The function $\alpha(q, x)$ is given by,

$$
\alpha(q, x)=1-x F_{1}\left(s^{*}(q)\right)-(1-x) F_{0}\left(s^{*}(q)\right)
$$

Among other properties, Lemma A.1 in the Appendix proves that $\alpha(q, x)$ strictly increases in $q$ and $x$. Since the ex-ante utility of the manager is the unconditional probability the board

\footnotetext{
${ }^{16}$ As long the bias of the manager is significant, non-verifiable information cannot be revealed in equilibrium when $m_{1}=\phi$. If $\tau$ is not a sufficient statistic for $\omega$ with respect to $\xi$ then non-verifiable information cannot be revealed even if $m_{1} \neq \phi$.
} 
approves the project, it can be written as follows,

$$
\begin{array}{r}
\mathbb{E}\left[u_{M}(e)\right]=e p+e(1-p)\left[\lambda \alpha(q(e), 0)+(1-\lambda) \cdot 1_{\left\{q(e) \geq \frac{1}{2}\right\}}\right] \\
+(1-e)\left[\lambda \alpha(q(e), p)+(1-\lambda) \cdot 1_{\left\{q(e) \geq \frac{1}{2}\right\}}\right]
\end{array}
$$

There are three terms in (2) to consider. The first term corresponds to events in which the manager successfully collects information about $\omega$ and finds out that $\omega=1$. Under those circumstances the manager can guarantee the approval of the project by sharing this verifiable information with the board. The second and the third terms correspond to the events in which the manager does not disclose information. In the second term the manager learns that $\omega=0$ and strategically withholds this information from the board. Conditional on this event the manager believes that the informed board will approve the project with probability $\alpha(q(e), 0)$ while the uninformed board will approve the project with probability one if $q(e) \geq \frac{1}{2}$ and reject it otherwise. In the third term the manager fails to collect hard evidence on $\omega$ and since non-verifiable information cannot be credibly communicated, no information is disclosed.

Lemma 2 Given learning intensity e, the manager's expected utility increases in $\lambda$ if and only if $q(e)<\frac{1}{2}$.

Lemma 2 reveals that despite his conflict of interests with the board (and shareholders), the manager benefits from high level of board's expertise exactly when this conflict of interests is significant, that is, when ex-ante the investment is non-profitable. Intuitively, when $q(e)<\frac{1}{2}$ the board approves the project conditional on non-disclosure only if it receives sufficiently high signal on the profitability of investment. An expert board is more likely to receive a high signal than an informed board. Thus, the manager benefits from the board's expertise. When $q(e)>\frac{1}{2}$ this dynamic is reversed and the board approves the project conditional on nondisclosure unless it receives sufficiently low signal on $\omega$. Thus, the board's expertise harms the 
manager.

The next proposition verifies that an equilibrium of the entire game exists and characterizes the manager's learning intensity in equilibrium.

Proposition 1 An equilibrium always exists. Let $e^{*}$ be the learning intensity in equilibrium and $q_{\min } \equiv \arg \min _{q \in[q(\bar{e}), p]}\left\{\frac{1-\alpha(q, q)}{1-q}\right\}$, then:

(i) If $p<\frac{1}{2}$ then $e^{*}$ decreases in $\lambda, e^{*}(\lambda=0)=\bar{e}$, and $e^{*}(\lambda=1)<\bar{e} \Leftrightarrow q_{\min }>q(\bar{e})$.

(ii) If $p \geq \frac{1}{2}$ there exist $e^{+}<\bar{e}$ and $\lambda^{+}(p, \bar{e})>0$ (where $\lambda^{+}<1 \Leftrightarrow q_{\min }<\frac{1}{2}$ ) such that:

$$
\begin{aligned}
& \text { - If } \lambda \leq \lambda^{+} \text {then } e^{*}=e^{+} . \\
& \text {- If } \lambda>\lambda^{+} \text {then } e^{*}>e^{+} \text {and } e^{*} \text { decreases in } \lambda .
\end{aligned}
$$

Proposition 1 reveals that the manager may choose to remain uninformed $\left(e^{*}<\bar{e}\right)$ even though learning does not impose direct costs on the manager. The reason is that being informed imposes indirect costs on the manager in the following sense. According to Lemma 1, the manager discloses information about $\omega$ if and only if $\omega=1$. Thus, the board infers from nondisclosure that either the manager failed to collect information about $\omega$ or that the manager strategically conceals information about $\omega=0$. The relative likelihood of the latter event increases with the amount of resources the manager spends on collecting information. Since the board observes the manager's learning intensity, it will adjust downward its posterior beliefs about $\omega=1$. This prejudice effect of learning reduces the likelihood the board approves the project, and hence, imposes an indirect cost on the manager. In other words, the indirect cost of learning is giving up the option to conceal information about $\omega=0$. The optimal learning intensity of the manager trades off this indirect cost with the possibility of learning that $\omega=1$ and using this information to persuade the board to approve the project. 
In order to gain more intuition, suppose $p<\frac{1}{2}$ and note that $(2)$ can be rewritten as

$$
\mathbb{E}\left[u_{M}(q)\right]=1-(1-p)\left[\frac{1-\lambda \alpha(q, q)}{1-q}\right]
$$

The manager's expected utility is uniquely determined by $q$, the posterior beliefs that $\omega=1$ conditional on non-disclosure. His decision on the learning intensity is equivalent to choosing $q \in[q(\bar{e}), p]$. The term $\lambda \alpha(q, q)$ is the ex-ante probability (prior to observing the signal about $\omega)$ the manager ascribes to the event that the board approves the project conditional on nondisclosure. It follows from (3) that if $\bar{e}=1$ then the manager chooses $q>0$ (or equivalently $e<1)$ if and only if there exists $q \in[0, p]$ such that $\lambda \alpha(q, q)>q$. Intuitively, $\lambda \alpha(q, q)$ is the alternative and indirect cost of collecting an extra unit of information, and $q$ is the likelihood that $\omega=1$ and hence the benefit from learning. As long as $\lambda \alpha(q, q)$ is greater than $q$, the manager gains from not collecting all the available information. The requirement that $q_{\min }>q(\bar{e})$ generalizes the condition above for any $\bar{e}$.

Proposition 1 also highlights that the equilibrium level of the manager's learning intensity almost everywhere decreases with the board's expertise, which is captured by $\lambda$. When $p<\frac{1}{2}$ and in the absence of disclosure, the board rejects the project unless its private information is sufficiently positive. Thus, all else equal, the likelihood the board approves the project increases with its expertise $\lambda$. This implies that in this region the indirect cost of learning increases with the board's expertise, but the benefit is fixed. Therefore, the manager has fewer incentives to learn about $\omega$. When $p \geq \frac{1}{2}$ the dynamic is partly reversed. The board approves the project unless its private information is sufficiently negative. In this case, the indirect cost of learning decreases with the board's expertise. Nevertheless, when $e$ is sufficiently high and $q(e)<\frac{1}{2}$, similar to description above for the case $p<\frac{1}{2}$, the indirect cost of learning will start to increase with the board's expertise. As Figure 2 illustrates, $e^{*}$ can be non-monotonic in $\lambda$ when $p \geq \frac{1}{2}$. 

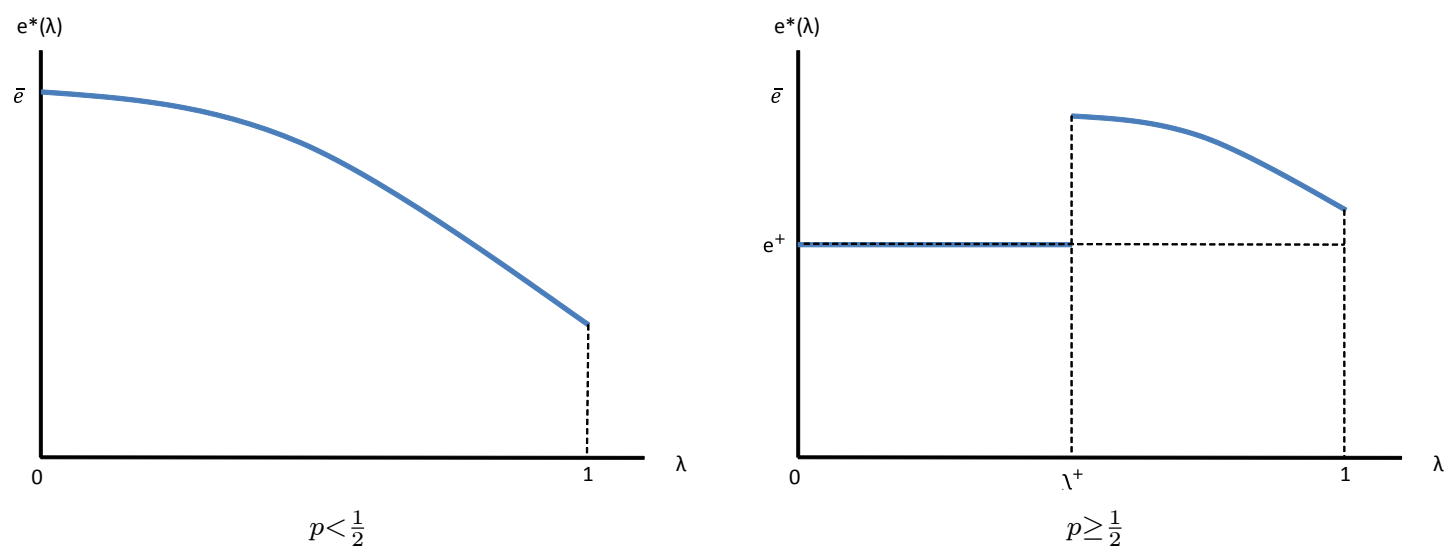

Figure 2 - Managerial learning intensity in equilibrium

The analysis demonstrates that the equilibrium level of learning intensity increases with $p$ almost everywhere. This is intuitive since higher $p$ implies that for a given level of learning intensity the likelihood the manager learns that $\omega=1$ is higher. In other words, the net return from collecting information increases with $p$. Nevertheless, the equilibrium level of $e$ can fall discretely at $p=\frac{1}{2}$. Indeed, when $\lambda$ is small and $p<\frac{1}{2}$ the board is likely to reject the project in the absence of additional information. Thus, the manager has strong incentives to collect and share information. However, when $\lambda$ is small and $p=\frac{1}{2}$ the board is likely to approve the project even without receiving additional information. Therefore, the incentives of the manager to collect information about $\omega$ are weaker.

While the manager's learning intensity in equilibrium does not necessarily increase with $p$, the probability the board approves the project in equilibrium does. Recall that the probability the board approves the project is exactly the manager's expected utility. As $p$ increases, it is easier for the manager to persuade the board to approve the project even if the learning intensity is lower. Corollary 1 concludes this observation and characterizes the effect of the board's expertise on the likelihood that the project is undertaken in equilibrium.

Corollary 1 The probability the project is approved in equilibrium strictly increases in $p$, and 
it strictly increases in $\lambda$ unless $p \geq \frac{1}{2}$ and $\lambda<\lambda^{+}$.

Interestingly, it follows from Corollary 1 that a higher board's expertise does not necessarily reduces the likelihood that the project is approved. One might expect that an expert board would be able to counteract the manager's bias toward the project's approval. However, Corollary 1 suggests that the board fails to do so exactly when a priori shareholders and the

manager disagree on the benefit from the project, that is, when $p<\frac{1}{2}$. This, however, does not necessarily imply a greater inefficiency. An informed board might act based on its private information which may contrast the available public evidence. Nevertheless, I demonstrate below that higher expertise can in fact harm shareholders even though the board's objective is to maximize their value.

\section{Shareholder Value}

Unlike the manager, shareholders derive value from the investment in the project if and only if $\omega=1$. Thus, their expected welfare is exactly the probability that $a=\omega$. Explicitly,

$$
\begin{aligned}
V(e, \lambda)= & p e+p(1-e)\left[\lambda \alpha(q(e), 1)+(1-\lambda) \cdot 1_{\left\{q(e) \geq \frac{1}{2}\right\}}\right] \\
& +(1-p)\left[1-\lambda \alpha(q(e), 0)-(1-\lambda) \cdot 1_{\left\{q(e) \geq \frac{1}{2}\right\}}\right]
\end{aligned}
$$

The first and second terms in (4) correspond to scenarios in which the board chooses $a=1$ conditional on $\omega=1$. This would be the case if either the board discloses information that $\omega=1$ or if conditional on non-disclosure the board finds it optimal to approve the project. The third term corresponds to scenarios in which the board chooses $a=0$ conditional on $\omega=0$. Since the manager never discloses information about $\omega=0$, the project is rejected only if information is not disclosed and the board finds it optimal to reject the project. 
Lemma 3 Shareholders' value $V(e, \lambda)$ strictly increases in e and $\lambda$.

It follows that shareholders' value increases with the amount of information that either their manager or their board obtains. This is intuitive since in both cases the probability that the "correct" action is taken is higher. Indeed, since the manager's disclosure policy is strategic, whether or not the manager discloses information, all else equal, more information is revealed if the manager spends more resources on learning. Therefore, the value-maximizing board is more likely to make the right decision. Similarly, all else equal, a better informed board is more likely to take the right decision ex-post, and shareholders are better off.

The next proposition demonstrates that despite the positive effect of $\lambda$ on $V(e, \lambda)$, and because the manager's optimal learning intensity can decrease with $\lambda$, the board's expertise might actually harm shareholders' value.

Proposition 2 Let $\lambda^{*}$ be the (highest) level of board's expertise that maximizes the value of shareholders in equilibrium.

(i) If $p<\frac{1}{2}$ there exists $\underline{e}<1$ such that $\lambda^{*}<1$ for all $\bar{e} \in[\underline{e}, 1]$ if and only if there exists $q \in(0, p]$ such that $\alpha(q, q)>q$.

(ii) If $p \geq \frac{1}{2}$ then $0<\lambda^{+} \leq \lambda^{*}$.

Proposition 2 shows that when the manager has sufficient access to information ( $\bar{e}$ is high), the expertise of directors can be harmful, especially when the conflict between shareholders and the manager is a priori significant. To see the intuition, recall the manager trades off two forces. On the one hand, the manager benefits from collecting information since this way he can persuade the board, regardless of its type, to approve the project if the evidence is supportive. On the other hand, there is a risk that the evidence is not favorable. In this case, despite the manager's attempt to conceal this information, the board would make the right inference and 
some of this information, but not all of it, is revealed. This increases the likelihood the board would reject the project and hence discourages the manager's incentives to collect information. As I explain below, the board's expertise changes this tradeoff in a non-trivial way.

Suppose ex-ante shareholders and the manager disagree on the benefit from taking the project, that is, $p<\frac{1}{2}$. If $\alpha(q, q) \leq q$ for all $q \in[0, p]$ then the board on average over-rejects the project and the direct benefit from learning always exceeds its alternative cost. Therefore, regardless of the board's expertise, the manager spends as many resources as possible on learning and $e^{*}=\bar{e}$. Since the manager's learning intensity is fixed at its highest level, it follows from Lemma 3 that shareholders' value increases with the expertise of their board. However, if there exists $q \in(0, p]$ such that $\alpha(q, q)>q$ then the expert board sometimes over-approves the project. Therefore, the alternative cost of learning increases with the board' expertise and the manager has fewer incentives to collect information. That is, the presence of expertise on the board discourages managerial learning and cooperation. If the manager has sufficient access to information $(\bar{e} \in[\underline{e}, 1])$, the loss of this information is relatively more important than the expertise of the board, and shareholder value would decrease with the board's expertise. In the Appendix I show that when $\bar{e}=1$ then a completely uninformed board can be optimal $\left(\lambda^{*}=0\right)$.

Alternatively, suppose shareholders and the manager a priori agree on the benefit from the project, that is, $p \geq \frac{1}{2}$. With low expertise $\left(\lambda<\lambda^{+}\right)$and in the absence of additional information it is unlikely the board would reject the project. Hence, the manager has very little incentives to collect information and thereby taking the risk he would find evidence against the project, evidence that he would have to conceal. With high expertise, however, it is no longer guaranteed that the board would approve the project in the absence of hard evidence in its support. Therefore, the manager has stronger incentives to collect information and the board's expertise benefits shareholders $\left(\lambda^{*} \geq \lambda^{+}\right)$. In the Appendix I give conditions on $\alpha(q, q)$ which guarantee $\lambda^{*}<1$ when $p \geq \frac{1}{2}$. So, even in this case shareholders value is not monotonically 
increasing with the board's expertise.

\section{Optimal Board Affiliation}

This section relaxes the assumption that the board is maximizing the value of shareholders. I characterizes the optimal level of board affiliation and show that the benefit to shareholders from appointing directors whose interests are misaligned depends on the expertise of these directors.

In order to study the optimal affiliation of the board I modify the baseline model and let the preferences of the board be $u_{B}(a, \omega)=v(a, \omega)+(1-2 \beta) \cdot 1_{\{a=1\}}$. Under these preferences the board approves the project if and only if it believes the probability that $\omega=1$ is greater than $\beta$. The parameter of interest in this section is $\beta .{ }^{17}$ Relative to shareholders' first best,

if $\beta>\frac{1}{2}$ then the board over-rejects the project and if $\beta \leq \frac{1}{2}$ the board over-approves it. Since the manager is biased toward the approval of the project, in the former case the board is considered to be hostile to the manager and in the latter case it is friendly. In the baseline model of Section I, the board was assumed to be unbiased and independent, that is, $\beta=\frac{1}{2}$.

In what follows it will become apparent that choosing $\beta \notin(0,1)$ is always sub-optimal from shareholders' point of view. Restricting attention to $\beta \in(0,1)$ is also consistent with the view that the board's characteristics matter only in the absence of cooperation between the manager and the board. Indeed, if $\beta \in(0,1)$ and the manager discloses information about $\omega$ the board defers to the manager's view regardless of its expertise and bias. However, if the manager does not disclose information the board approves the project if and only if the board is uninformed and $q \geq \beta$, or the board is informed and its private signal $s$ is greater than $s^{*}(q, \beta) \equiv l^{-1}\left(\frac{\beta}{1-\beta} \frac{1-q}{q}\right)$.

For a given level of posterior beliefs, the probability that the board approves the project

\footnotetext{
${ }^{17}$ Throughout the analysis it is implicitly assumed that the board's characteristics $\beta$ and $\lambda$ are independent of each other. A correlation between $\beta$ and $\lambda$ may create a non-trivial trade-off which is partly left out from the present analysis.
} 
decreases with $\beta$. Since the manager's sole objective is to maximize the probability that the project is approved, the manager's expected utility in equilibrium decreases with $\beta$. Yet, taking power from the opportunistic manager does not necessarily mean that shareholders are better off. A biased board makes ex-post inefficient decisions. Unless the board's bias changes the incentives of the manager to collect information and then share it with the board, shareholders would be worse off . Therefore, a biased board can benefit shareholders only if consequently the manager is incentivized to collect more information than otherwise.

Let us define by $\beta^{*}$ the bias of the board that maximizes the ex-ante value of shareholders and is closest in absolute terms to $\frac{1}{2}$. The next proposition characterizes $\beta^{*}$.

\section{Proposition 3}

(i) For any $\lambda \in[0,1]$ there is $e_{\lambda}<1$ such that $\beta^{*} \geq \frac{1}{2}$ for all $\bar{e} \in\left[e_{\lambda}, 1\right]$.

(ii) There are $\bar{\lambda} \geq 0$ and $\lambda^{+}>0$ such that if and only if $\lambda>\bar{\lambda}$ or, $p \geq \frac{1}{2}$ and $\lambda<\lambda^{+}$, then $\beta^{*}>\frac{1}{2}$ for all $\bar{e} \in\left[e_{\lambda}, 1\right]$.

Proposition 3 demonstrates that when the manager has sufficient access to information $(\bar{e}$ is high), a bias can be optimal only if the bias of the board is against the manager. That is, the board is biased toward rejecting the project. As was mentioned in the introduction, this result is different from the existing literature on optimal board structure that builds on the advisory role of the board. This literature argues that in order to facilitate meaningful boardroom discussion (cheap talk a la Crawford and Sobel (1982)), directors should be affiliated with the management. In other words, an optimal board structure implies that the board is biased toward the manager. By contrast, the present analysis highlights that shareholders obtains the highest valuation when directors inherently take the opposite view from the management. In this respect, the result has the spirit of Dewatripont and Tirole (1999) who show that a benefit of creating advocates within organizations is the generation of information on alternative 
policies. ${ }^{18}$

How does a stronger misalignment of interests between the board and the manager facilitate cooperation? To see the intuition, recall that if the manager collects supportive evidence for the project the board can be convinced to approve the project regardless of its characteristics. As the board becomes more biased against the project, the manager realizes that the only realistic way to convince the board to approve the project is by providing these hard evidence. Thus, when information is hard, a wider gap between the preferences of the manager and the board can in fact facilitate collection and revelation of information.

When the manager has sufficient access to information ( $\bar{e}$ is high), the benefit for shareholders from having a more informative decision making outweighs the possibility that the board would reject profitable projects. In the Appendix I demonstrate that when $\lambda>0$ and $\bar{e}<1$ (and $\bar{e}$ can be arbitrarily close to one) the cost of biasing the board can be significant and therefore $\beta^{*}$ is bounded away from one, yet strictly above $\frac{1}{2}$.

Proposition 3 also highlights the interaction between the board's bias and its expertise. Biasing the board away from maximizing shareholders' value is optimal when the board's expertise is high $(\lambda>\bar{\lambda}) \cdot{ }^{19}$ Intuitively, as long as $e<1$ the expert board approves the project with a strictly positive probability. Thus, when the board is unbiased, the manager exploits this opportunity by keeping the learning intensity relatively low. As was discussed above, biasing the board against the project increases the incentives of the manager to collect and share information, and thus, the gains to shareholders from having this bias are relatively large when the board has expertise. Similarly, when $p \geq \frac{1}{2}$ but the expertise of the board is low $\left(\lambda<\lambda^{+}\right)$, the unbiased board is likely to approve the project even without any supportive evidence. Thus, the gains from the biasing the board against the project are large in this case as well.

\footnotetext{
${ }^{18}$ See also Landier, Sraer, and Thesmar (2009) and Kamenica and Gentzkow (2011) for related contributions.

${ }^{19}$ In the Appendix I show that $\bar{\lambda}<1$ if and only if $\max _{q \in\left(0, \min \left\{p, \frac{1}{2}\right\}\right)} \alpha(q, q)>q$ and that if $\lim _{q \rightarrow 0} \frac{\partial \alpha(q, q)}{\partial q}=\infty$ then $\bar{\lambda}=0$.
} 


\section{Board's Reputation Concerns For Expertise}

When shareholders have influence on the composition of the board, directors may have incentives to develop reputation for being experts. This section studies the effect of board's reputation concerns on the value of the firm, and its interaction with boardroom transparency.

\section{A Modified Setup}

In order to study reputation concerns I first reinterpret the baseline model. The board can be of two types, $\rho \in\{0,1\}$. The layman board $(\rho=0)$ is perfectly uninformed about $\omega$, whereas the expert board $(\rho=1)$ privately observes signal $s$ on $\omega$. The expertise of the board $\rho$ is the board's private information, but shareholders and the manager share the common prior that the board is expert with probability $\lambda \in[0,1]$.

The baseline model is also modified by augmenting the board's preferences with concerns for reputation. In particular, $u_{B}(a, \omega)=v(a, \omega)+\Gamma(\operatorname{Pr}[\rho=1 \mid \mathcal{F}])$ where $\operatorname{Pr}[\rho=1 \mid \mathcal{F}]$ is shareholders' beliefs that the board is an expert conditional on the available public information denoted by $\mathcal{F}$. The function $\Gamma(\cdot)$ is the board's direct utility from reputation and it is strictly increasing and continuous. I normalize $\Gamma(\cdot)$ by setting $\Gamma(0)=0$. Furthermore, I assume that career concerns do not entirely override the board's fiduciary duty to maximize its shareholders' value. Below I show that $\Gamma(1)<\bar{\Gamma} \in(0,1]$ guarantees the existence of equilibrium.

Shareholders form their beliefs on the board's expertise solely based on its decision to approve the project. They do not observe how much information the manager collects or shares with the board. Later, I relax this assumption and study the effect of transparency. I also focus on shareholders' interim beliefs and assume that they do not observe the outcome of the project before they form their expectation, that is, $\mathcal{F}=\{a\}$. Presumably, it takes a while until the return on the investment is realized. ${ }^{20}$

\footnotetext{
${ }^{20}$ While $a \in \mathcal{F}$ is necessary for our results, $1_{\{\omega=a\}} \notin \mathcal{F}$ is not. As Lemma 5 shows, if $a \in \mathcal{F}$ then concerns for reputation distort the board's decision in equilibrium. This distortion is key for our results, and in the Appendix I show that it exists in equilibrium even if $\left\{1_{\{\omega=a\}}, a\right\} \subset \mathcal{F}$.
} 
In order to focus on the effect of reputation I simplify the information structure of the baseline model as follows. First, the manager has full access to information, $\bar{e}=1$. Second, the expert board privately observes a binary signal $s \in\{0,1\}$ where $\operatorname{Pr}[s=\omega \mid \omega]=\pi$ and $\pi \in(1 / 2,1)$ is the precision of the board's signal. Similar to Section I, I assume that $1-\pi<$ $p<\pi$ in order to ensure that the board's expertise can add value in the absence of additional information. Importantly, all the results of the previous sections hold under the binary setup as well.

\section{B Analysis of Reputation Concerns}

While shareholders do not observe how much information the manager collects and shares with the board, in equilibrium, they have correct expectations. At the same time, how shareholders form their beliefs about the board's expertise affects the board's decision to approve the project, and thereby the manager's incentives to collect and share information. A solution for this fixed point problem is not always guaranteed, and an equilibrium may not exist if the board weighs its reputation too heavily.

For example, suppose shareholders believe that an expert board is more likely to reject the project than a layman board. If the board is very sensitive to its reputation then regardless of its expertise the board has incentives to take the action that maximizes its reputation. Thus, the board would reject the project even if approving it benefits shareholders. The board's behavior is therefore inconsistent with shareholders' beliefs. At the same time, if shareholders believe that the expert and the layman board follow the same decision rule, as long as the board put positive weight on shareholders' value, both the expert and the layman board have strict incentives to take the action that maximizes shareholders' value conditional on their private information. Because their endowment of information is heterogenous, the layman and the expert board would behave differently. Once again, this would be inconsistent with shareholders' expectations. All in all, an equilibrium of the game may not exist. 
The next lemma provides a sufficient condition for the existence of equilibrium. Hereafter the analysis implicitly assumes that this condition is met.

Lemma 4 For any $(p, \lambda, \pi)$ there exists $\bar{\Gamma} \in(0,1]$ such that if $\Gamma(1) \leq \bar{\Gamma}$ an equilibrium exists.

In principle, there may exist equilibria of the game which involve off-equilibrium events. I restrict these events as follows. First, I require that off-equilibrium beliefs are credible in the sense of Grossman and Perry (1986). Second, shareholders' beliefs about the board's expertise are bounded away from zero: whenever it is optimal for the layman board to deviate to an off-equilibrium action, there is a strictly positive probability that the expert board finds it optimal to deviate as well. That is, if the layman board deviates and approves (rejects) the project, the expert board with signal $s=1(s=0)$ must find it optimal to deviate the same way as well. For this reason I assume that the off-equilibrium beliefs cannot lie below these lower bounds. Lemma A.9 in the Appendix shows that under these two restrictions, there are no off-equilibrium events within this class of equilibria.

To solve for equilibria of the game I start with the observation that parts (i) and (ii) of Lemma 1 continue to hold under the modified setup, and hence the functional form of $q(e)$ does not change. Part (iii) of Lemma 1, however, changes. Consider two cases. First, suppose the manager discloses verifiable information about $\omega$. Regardless of its expertise, the board updates its posterior beliefs to $\operatorname{Pr}[\omega=1]=m_{1}$, where $m_{1}=\omega$. The board approves the project if and only if $m_{1} \geq \frac{1+\Delta}{2}$, where $\Delta \equiv \Gamma(\operatorname{Pr}[\rho=1 \mid a=0])-\Gamma(\operatorname{Pr}[\rho=1 \mid a=1])$ is the board's reputation gains from rejecting the project. Note that $\Delta$ is independent of the board's actual expertise and it is determined in equilibrium. Thus, $\Delta$ is fixed from the board's point of view. The assumptions $0=\Gamma(0)<\Gamma(1)<1$ guarantee that $|\Delta|<1$ and hence the unique response of the board is to approve the project if and only if $m_{1}=1$. Similar to Lemma 1 , the board defers to the manager's view when the latter shares information, and in this case, concerns for 
reputation do not distort the board's behavior.

By contrast, when the manager does not share information, the board forms its beliefs based on its expertise and the inference from the manager's decision not to share information. The board approves the project if and only if $\frac{l(s)}{l(s)+(1-q(e)) / q(e)} \geq \frac{1+\Delta}{2}$. If in equilibrium the expert board is more likely to reject (approve) the project than the layman board, then $\Delta>0$ $(\Delta<0)$ and reputation concerns for expertise distort the board's decision toward over-rejection (over-approval) of the project. Taking into account the manager incentives to learn and share information, the next lemma characterizes this distortion.

Lemma 5 Conditional on non-disclosure, the board over-rejects the project $\left(\Delta^{*}>0\right)$ in equilibrium if and only if $q\left(e^{*}\right)>\frac{1}{2}$.

Lemma 5 suggests that in order to create the impression that board members have private information about the prospects of the project, the layman board imitates the behavior of the expert board. In particular, in any equilibrium shareholders correctly anticipate the managerial learning intensity and $q\left(e^{*}\right)$. From shareholders' point of view, an expert board seemingly ignores useful public information. It rejects projects which a priori seem profitable, and vis-a-versa. Nevertheless, this behavior is optimal if the board's private information is sufficiently precise, $1-\pi<p<\pi$. In order to create the impression that the board is in fact an expert, the board under-reacts to the public information conditional on non-disclosure: it over-approves the project when the posterior belief conditional on non-disclosure $q\left(e^{*}\right)$ is lower than $\frac{1}{2}$, and over-rejects its when $q\left(e^{*}\right) \geq \frac{1}{2}$. This result is similar in spirit to Prendergast and Stole (1996) who demonstrate that investment distortions arise from managers attempting to influence perceptions of their expertise by exaggerating their private information.

In what follows I argue that the manager can exploit the distortion in the board's decision making, and hence, the main channel through which the board's concerns for reputation affect 
shareholders' value is by changing the manager's incentives to cooperate with the board.

Proposition 4 In equilibrium, concerns for reputation harm the value of shareholders if and only if $\lambda<\lambda^{\dagger}$ where

$$
\lambda^{\dagger} \equiv \begin{cases}\frac{1}{\pi(3-2 \pi)} & \text { if } p \geq \frac{1}{2} \\ \frac{1}{2 \pi} & \text { if } p<\frac{1}{2}\end{cases}
$$

Moreover, concerns for reputation increase the likelihood of project's approval unless $p \geq \frac{1}{2}$ and $\lambda<\lambda^{\dagger}$.

Proposition 4 compares between games with and without reputation concerns. To see the intuition behind the proposition, suppose first that $\lambda \geq \lambda^{\dagger}$. The likelihood the board is an expert is relatively high. In order to maximize the probability the project is approved by the board, the manager collects information such that the expert board is more likely than the layman board to approve the project conditional on non-disclosure. The approval of the project signals shareholders that the board is an expert and shareholders accordingly update upward their beliefs about its expertise. Thus, reputation concerns create additional benefits for the board to approve the project, even if it does not add value to shareholders. The manager anticipates this distortion and adjusts his learning intensity: he can collect more information and at the same time keep the probability of the project's approval conditional on non-disclosure unchanged. Similar to Section II, the board's bias can boost the incentives of the manager to collect and share information and thereby benefit shareholders. Different from Proposition 3, however, in this section the bias benefits shareholders when it is toward the manager and not against him. Indeed, Proposition 3 does not rule out the possibility that a bias toward the manager $\left(\beta<\frac{1}{2}\right)$ would benefit shareholders more than an unbiased board $\left(\beta=\frac{1}{2}\right)$ would. Instead, it proves that for any bias toward the manager there is a level of bias against the manager which yields even a higher value for shareholders, and therefore the 
optimal bias $\beta^{*}$ is always against the manager. In the current section, the bias in the board's behavior is determined endogenously by reputation concerns for expertise, and the equilibrium put constraints on which bias can arise endogenously.

Reputation concerns can also have adverse effect on welfare. For example, suppose $\lambda<\lambda^{\dagger}$ and $p<\frac{1}{2}$. As in Section I, when reputation concerns are absent the board is unlikely to approve the project unless the manager cooperates. In equilibrium, the manager has strong incentives to collect and share information, and the board makes decisions based on the manager's report rather than on its expertise. With reputation concerns, however, the manager has fewer incentives to collect information. By forcing the board to make decisions based on its expertise, the manager boosts the board's incentives to approve the project. Indeed, all else equal, when $p<\frac{1}{2}$ the expert board is more likely to approve the project conditional on non-disclosure. Therefore, the approval of the project signals shareholders high expertise, and both types of the board have additional private benefits to approve the project. Overall, not only the board over-approves the project, less information is exchanged in the boardroom. Importantly, it is the indirect effect on the manager's incentives to collect and share information that dominates and plays the key role in the welfare analysis. ${ }^{21}$

Proposition 4 suggests that in most cases the manager's ability to persuade the board to approve the project improves when the latter is subject to reputation concerns. By controlling the amount of information and exploiting the board's sensitivity to shareholders' beliefs, the manager can strategically sway the board toward the project's approval. In this sense, the friendliness of the board is endogenous. Since the interaction between shareholders and the manager is not a zero-sum game, this increase in "managerial power" does not necessarily come on the expense of shareholders. Nevertheless, Proposition 4 shows that there are circumstances

\footnotetext{
${ }^{21}$ The direct effect of board's reputation concerns can be measured as the change in shareholders' welfare if one adds reputation concerns to the analysis while keeping the learning intensity on its equilibrium level in a game without reputation concerns. In the Appendix I show that under most circumstances, and in particular when $\lambda<\lambda^{\dagger}$, this direct effect is zero.
} 
where the board's concerns for reputation transfer welfare from shareholders to their manager. ${ }^{22}$

Finally, note that when the expertise of the board is believed to be low $\left(\lambda<\frac{1}{2}\right)$ or when the uncertainty about it is high $\left(\lambda \approx \frac{1}{2}\right)$, concerns for reputation should matter the most. Nevertheless, since $\lambda^{\dagger}>\frac{1}{2}$, Proposition 4 implies that under those circumstances putting more pressure on the board (and thereby increasing its awareness for reputation) would achieve exactly the opposite outcome. This result challenges the idea that giving more power to shareholders in determining the identity of directors is always beneficial.

\section{Boardroom Transparency}

The availability of public information can change the perception of shareholders (and other market participants) on the expertise of directors. With more information, shareholders can better judge the board's performances and consequently change the way they form their beliefs. Thus, when board members are concerned about their reputation, the transparency of the interaction in the boardroom matters.

In order to explore the effect of boardroom transparency I assume hereafter that the manager's message to the board is public and hence observable by shareholders. That is, if the manager discloses information to the board $\left(m_{1}=\omega\right)$ shareholders observe this message as well. If the manager chooses not to disclose information $\left(m_{1}=\phi\right)$, then shareholders know that no information was disclosed.

Proposition 5 Suppose equilibrium exists with and without transparency in the boardroom.

(i) Transparency reduces the amount of (ex-ante) disclosure in equilibrium if and only if $p \geq \frac{1}{2}$ and $\lambda<\lambda^{\dagger}$.

(ii) Transparency amplifies the effect of reputation on shareholder value unless $p<\frac{1}{2}$ and

\footnotetext{
${ }^{22}$ Managerial power is measured by $\operatorname{Pr}[a=1]$. In the Appendix I show that similar results hold if one measures managerial power by $\operatorname{Pr}[a=1 \mid \omega=0]$.
} 


$$
\lambda<\lambda^{\dagger}
$$

Proposition 5 demonstrates that transparency may have adverse effects even when the nature of information is not proprietary. The observability of message $m$ changes the way shareholders form their beliefs on the board. To understand how, recall that upon disclosure the expertise of the board plays no role and the board always finds it optimal to defer to the manager's view. ${ }^{23}$ With transparency, shareholders can tell whether the board approves the project based on the manager's recommendation or based on its own expertise. This feature magnifies the effect of reputation and increases the board's gains or losses from reputation. Overall, the board's endogenous bias caused by its concerns for reputation increases with transparency. According to Proposition 4 and the discussion that follows it, this bias may have positive and negative effects on the manager's incentives to collect and disclose information, and thereby on the value of shareholders. Therefore, by magnifying the effect of reputation, boardroom transparency can decrease the amount of information that is exchanged in the boardroom and harm welfare.

\section{Concluding Remarks}

This paper studies the optimal structure of the board by focusing on the expertise of its directors. I show that the expertise of the board can diminish the incentives of the manager to collect and share information, and thereby harm shareholder value. The paper also argues that an optimal board structure should consist of dissenting directors, and that directors' reputation concerns can shift power from the board to the manager on the expense of shareholders.

The welfare analysis in the paper demonstrates that the adverse effect of the board's expertise depends on the importance of this expertise relative to the manager's input. This observation has important empirical implications. It suggests that if the expertise of directors

\footnotetext{
${ }^{23}$ Since shareholders observe the board's action, the analysis does not change if instead shareholders know when information is disclosed but they do not observe the content of the message.
} 
complements the manager's knowledge it would add value to shareholders, but if it substitutes the manager's knowledge then directors' expertise can actually destroy value. Thus, when measuring the value of the expertise of the board, it is important to distinguish between these properties. For example, politically-connected directors are likely to add value to shareholders of a defense contractor, while directors with financial expertise might actually destroy value in these companies if the CEO herself has a strong background in finance. Similarly, venture capital funds, which often take board seats in their portfolio companies, are likely to add value to these start-up companies whenever they complement the founder's technological expertise with the marketing and management know-how. The model predicts that despite the technological knowledge that some partners in venture capital funds might have, a tendency to intervene based on this type of expertise might in fact harm the value of the venture.

The adverse effect of the board's expertise also depends on the magnitude of the conflict of interests between the manager and shareholders. If the manager is sufficiently aligned with the interests of shareholders, it is straight forward that any kind of expertise on the board would (weakly) enhance the value of the firm. However, the analysis in this paper emphasizes that low expertise of directors can be an optimal arrangement from shareholders' point of view even if the manager is significantly biased. This prediction differs from stories which build on the ability of the CEO to appoint directors, in which case, low expertise on the board is associated with a lower shareholder value.

The analysis also provides justification for the appointment of dissenting directors in corporate boards. Inherent dissent on the board can be useful since it enhances the incentive of the manager to collect and share information. Sharing information leads to consensus and thereby more efficient decision making of the board. This observation suggests that the presence of stakeholders, other than shareholders, on corporate board can benefit shareholders. For example, hedge funds, employees, and creditors (but maybe suppliers and costumers as well) are likely to disagree with shareholders and the incumbent management on strategic issues such as 
takeovers and payout policy, but at the same time they may have a relevant expertise. This inherent disagreement, as the analysis suggests, can in fact facilitates more efficient decision making.

Finally, the analysis suggests that managers may exploit board members' desire to demonstrate expertise and thereby extract welfare from shareholders. The attention that directors devote to the public perception of their expertise is a plausible reflection of the recent rise in shareholder power. Indeed, with the growing popularity of majority voting in director elections and the increased shareholder attention to director qualifications and board composition, the re-election of directors is not automatic. The analysis highlights that this trend may have unintended and adverse consequences. This observation is somewhat consistent with the empirical evidence that the abnormal returns for firms announcing the adoption of majority voting instead of plurality voting for director elections are insignificant (Sjostrom and Kim (2007)). 


\section{References}

[1] Adams, Ren’ee B., and Daniel Ferreira, "A Theory of Friendly Boards," Journal of Finance, January 2007, 62 (1), 217-250.

[2] Alexander, Cindy R., Mark A. Chen, Duane J. Seppi, and Chester S. Spatt, (2008), "The Role of Advisory Services in Proxy Voting", NBER Working Paper No. w15143.

[3] Almazan, Andres, and Javier Suarez, 2003, "Entrenchment and severance pay in optimal governance structures", Journal of Finance 58, 519-547

[4] Baranchuk, Nina, and Philip H. Dybvig, 2009, "Consensus in diverse corporate boards", Review of Financial Studies 22, 715-747.

[5] Burkar, Mike, Denis Gromb, and Fausto Panunzi, 1997, "Large Shareholders, Monitoring and the Value of the Firm" Quarterly Journal of Economics 112, 693-728.

[6] Chakraborty, Archishman, and Bilge Yilmaz, 2011 "Authority, Consensus and Governance" Working paper University of Pennsylvania.

[7] Che, Yeon-Koo, and Kartik Navin, 2009. "Opinions as incentives", Journal of Political Economy 117 (5), 815-860

[8] Crawford, Vincent, and Joel Sobel, 1982, "Strategic Information Transmission", Econometrica 50:1431-51.

[9] Cremer, Jacques, 1995 "Arm's Length Relationships," Quarterly Journal of Economics, CX (2), 275-296.

[10] Defond, Mark, Rebecca Hann, and Xuesong Hu, 2005. "Does the market value financial expertise on audit committees of boards of directors?" Journal of Accounting Research 43, 153-193. 
[11] Dewatripont, Mathias, Ian Jewitt, and Jean Tirole, "The economics of career concerns, part I: Comparing information structures," Review of Economic Studies, 1999, 66 (1), $183-198$.

[12] Dewatripont, Mathias, and Jean Tirole, 1999, "Advocates", Journal of Political Economy $107,1-39$.

[13] Dye, Ronald A., 1990 "Mandatory Versus Voluntary Disclosures: The Cases of Financial and Real Externalities," Accounting Review, 1-24.

[14] Fich, Eliezer M., "Are Some Outside Directors Better than Others? Evidence from Director Appointments by Fortune 1000 Firms," Journal of Business, 2005, 78 (5), 1943-1971.

[15] Fisch Jill E., 2011 "The Destructive Ambiguity of Federal Proxy Access" Emory Law Journal Vol. 61, Pg. 435, 2012.

[16] Grossman, Sanford J., and Motty Perry 1986, "Perfect Sequential Equilibrium." J. Econ. Theory 39 (June 1986): 97-119.

[17] Grossman, Sanford. J., and Oliver. D. Hart 1986, "The Costs and Benefits of Ownership: A Theory of Vertical and Lateral Integration,” Journal of Political Economy, 94(4), 691-719.

[18] Guner, Burak, Ulrike Malmendierm, and GeoffreyTate , 2008. "Financial expertise of directors". Journal of Financial Economics 88, 323-354.

[19] Harris, Milton and Artur Raviv, 2005, "Allocation of decision-making authority," Review of Finance9, 353-383.

[20] Harris, Milton and Artur Raviv, 2008, "A Theory of Board Control and Size," Review of FinancialStudies, 21, 1797-1832.

[21] Harris, Milton and Artur Raviv, 2010, "Control of Corporate Decisions: Shareholders vs. Management," Review of Financial Studies 23, 4115-4147. 
[22] Hermalin, Benjamin E., and Michael S. Weisbach 2012 "Information Disclosure and Corporate Governance,". Journal of Finance, February 2012, 67 (1), 195-234.

[23] Hirshleifer, David, and Anjan V. Thakor, 1998 "Corporate Control Through Board Dismissals and Takeovers," Journal of Economics \& Management Strategy, 7(4), December, 489-520.

[24] Holmstrom, Bengt, 1999 "Managerial incentive problems: a dynamic perspective," Review of Economic Studies, 66 (1), 169-182.

[25] Kahan, Marcel, and Edward B. Rock, 2010 ,"Embattled CEOs", Texas Law Review, Vol. 88, No. 987 ,

[26] Kamenica, Emir and Matthew Gentzkow, 2011. "Bayesian Persuasion," American Economic Review, 101(6): 2590-2615.

[27] Landier, Augustin, David Sraer, and David Thesmar, 2009, "Optimal dissent in organizations", Review of Economic Studies 76, 761-794.

[28] Levit, Doron, 2011, "Advising Shareholders in Takeovers," Working paper, University of Pennsylvania

[29] Prat, Andrea, 2005, "The wrong kind of transparency". American Economic Review 95, $862-877$.

[30] Prendergast, Canice, and Lars Stole, 1996, "Impetuous youngsters and jaded old-timers: Acquiring a reputation for learning," Journal of Political Economy 104, 1105-1134.

[31] Rajan, Raghuram, 1992, "Insiders and Outsiders: The Choice between Informed and Arm's-Length Debt," Journal of Finance, XLVII (1992), 1121-40

[32] Ruiz-Verdu, Pablo, and Ravi Singh, 2011, "Board Reputation, CEO Pay, and Camouflaged Compensation," Working paper. 
[33] Sjostrom, William K., and Young Sang Kim, 2007, "Majority Voting for the Election of Directors," Connecticut Law Review 40, 459-510.

[34] Song, Fenghua and Anjan V. Thakor, 2006, "Information Control, Career Concerns, and Corporate Governance," Journal of Finance, August 2006, 61 (4), 1845-1896.

[35] Von Thadden, Ernst-Ludwig, 1995, "Long-term contracts, short-term investment and monitoring," Review of Economic Studies 62, 557-575. 


\section{Appendix}

The Appendix has three parts: Proofs for Sections I, II, and III. The supplemental results are collected in a separate appendix.

\section{A Proofs of Section I}

Lemma A.1 For any $q \in[0,1], p \in[0,1]$, and $\beta \in(0,1)$ let $s^{*}(q, \beta) \equiv l^{-1}\left(\frac{\beta}{1-\beta} \frac{1-q}{q}\right)$ and

$$
\alpha(q, p, \beta) \equiv 1-p F_{1}\left(s^{*}(q, \beta)\right)-(1-p) F_{0}\left(s^{*}(q, \beta)\right)
$$

The following holds: (i) $\alpha(q, p, \beta)=p \alpha(q, 1, \beta)+(1-p) \alpha(q, 0, \beta)$; (ii) $\alpha(q, p, \beta)=1 \Leftrightarrow q=1$ and $\alpha(q, p, \beta)=0 \Leftrightarrow q=0 ;$ (iii) $\alpha(q, p, \beta)$ is continuous and differentiable in all arguments; (iv) $\frac{\partial \alpha(q, p, \beta)}{\partial q}>0, \frac{\partial \alpha(q, p, \beta)}{\partial p}>0$, and $\frac{\partial \alpha(q, p, \beta)}{\partial \beta}<0$.

Proof. In order to simplify exposition we use $s^{*}$ for $s^{*}(q, \beta)$. Part $(i)$ (the linearity of $\alpha(q, p, \beta)$ in $p$ ) follows directly from expression (A1). Consider part (ii). Since $F_{\omega}$ strictly increases in $s$ and it is strictly positive for any $s>\underline{s}$, we get $\alpha(q, p, \beta)=1 \Leftrightarrow s^{*}=\underline{s}$. Given the definition of $s^{*}$ and the monotone likelihood ratio property (hereafter, MLRP), $s^{*}=\underline{s} \Leftrightarrow q=1$. A similar (and symmetric) argument proves that $\alpha(q, p, \beta)=0 \Leftrightarrow q=0$. Part (iii) follows from the continuity and differentiability of $F_{\omega}$ and $f_{\omega}$. Finally, to see part $(i v)$ note that $\frac{\partial \alpha(q, p, \beta)}{\partial q}=-\left[p f_{1}\left(s^{*}(q, \beta)\right)+(1-p) f_{0}\left(s^{*}(q, \beta)\right)\right] \frac{\partial s^{*}(q, \beta)}{\partial q}$ where $\frac{\partial s^{*}(q, \beta)}{\partial q}=\frac{-1}{\left.\frac{\beta}{1-\beta} q^{2} \frac{\partial l(s)}{\partial s}\right|_{s=s^{*}(q, \beta)}}<0$ due to the MLRP. It follows that $\frac{\partial \alpha(q, p, \beta)}{\partial q}>0$ for any $p \in[0,1]$ and $q>0$. Since $\alpha(0, p, \beta)=0$ and $\alpha(q, p, \beta) \in[0,1]$ is continuous and differentiable everywhere, $\left.\frac{\partial \alpha(q, p, \beta)}{\partial q}\right|_{q=0}>0$ as required. A similar argument shows that $\frac{\partial \alpha(q, p, \beta)}{\partial \beta}<0$. Finally, $\frac{\partial \alpha(q, p, \beta)}{\partial p}=F_{0}\left(s^{*}(q, \beta)\right)-F_{1}\left(s^{*}(q, \beta)\right)$ and the MLRP implies $F_{1}(s)<F_{0}(s)$ for any $s$. Overall, $\frac{\partial \alpha(q, p, \beta)}{\partial p}>0$ for any $q$.

Proof of Lemma 1. Consider first part (ii). Suppose on the contrary there are $m_{2}^{\prime} \neq m_{2}^{\prime \prime}$ 
and $x^{\prime} \neq x^{\prime \prime}$ in $I_{e}$ such that $\sigma_{e}^{*}\left(\phi, m_{1}^{\prime} \mid x^{\prime}\right)>0, \sigma_{e}^{*}\left(\phi, m_{2}^{\prime \prime} \mid x^{\prime \prime}\right)>0$, and $\operatorname{Pr}_{s}\left[a_{e,\left(\phi, m_{1}^{\prime}\right)}(s)>\right.$ $\left.a_{e,\left(\phi, m_{2}^{\prime \prime}\right)}^{*}(s)\right]>0$. Recall the manager is maximizing the probability that the project is approved by the board. Thus, for any $x$ in $I_{e}$ sending message $m_{2}^{\prime \prime}$ is strictly dominated by sending message $m_{1}^{\prime}$. This contradicts the assumption that $\sigma_{e}^{*}\left(\phi, m_{2}^{\prime \prime} \mid x^{\prime \prime}\right)>0$. Since any information about $\xi$ changes the board's posterior beliefs when $m_{1}=\phi$, and consequently its decision, information on $\xi$ cannot be revealed when $m_{1}=\phi$. Hereafter, without the loss of generality, I assume that message $m_{2}$ is ignored by the board.

Consider part (i) and suppose that $q(e)=q_{0}$. Conditional on $e$ and observing $\omega$, the manager's expected utility as a function of $\sigma$, the actual probability the manager chooses $m_{1} \neq \phi$, is given by

$$
\mathbb{E}\left[u_{M}(\sigma) \mid \omega\right]=(1-\sigma)\left[\lambda \alpha\left(q_{0}, \omega\right)+(1-\lambda) \cdot 1_{\left\{q_{0} \geq \frac{1}{2}\right\}}\right]+\sigma \omega
$$

Note that $q_{0}$ is independent of $\sigma$, and thus, $\frac{\partial \mathbb{E}\left[u_{M}(\sigma) \mid \omega\right]}{\partial \sigma}=\omega-\lambda \alpha\left(q_{0}, \omega\right)-(1-\lambda) \cdot 1_{\left\{q_{0} \geq \frac{1}{2}\right\}}$. Using Lemma A.1 part (ii), it follows that $\left.\frac{\partial \mathbb{E}\left[u_{M}(\sigma) \mid \omega\right]}{\partial \sigma}\right|_{\omega=1}>\left.\frac{\partial \mathbb{E}\left[u_{M}(\sigma) \mid \omega\right]}{\partial \sigma}\right|_{\omega=0}$. Therefore, in any equilibrium $q_{0} \leq p$. Since $q_{0} \leq p<1$, Lemma A.1 part (ii) also implies that $\left.\frac{\partial \mathbb{E}\left[u_{M}(\sigma) \mid \omega\right]}{\partial \sigma}\right|_{\omega=1}>0$. That is, the manager has strict incentives to disclose $\omega=1$. Note that $\left.\frac{\partial \mathbb{E}\left[u_{M}(\sigma) \mid \omega\right]}{\partial \sigma}\right|_{\omega=0} \leq 0$ and hence the manager has weak incentives to conceal $\omega=0$. While $\left.\frac{\partial \mathbb{E}\left[u_{M}(\sigma) \mid \omega\right]}{\partial \sigma}\right|_{\omega=0}=0$ is feasible (when $q_{0}=0$ or when $q_{0}<\frac{1}{2}$ and $\lambda=0$ ), playing mixed strategy at the disclosure stage cannot change the board's approval decision (which is the objective of the manager) or the managerial learning intensity in equilibrium. Therefore, without the loss of generality, $\sigma=\omega$ as required.

Part (iii) follows from parts (i) and (ii) of Lemma 1 and the discussion that precedes it. To show that $q(e, m) \equiv \frac{p-e p}{1-e p}$ note that,

$$
q(e, m) \equiv p \frac{\int g(\xi \mid \omega=1, e)[e \sigma(m \mid \omega=1, \xi)+(1-e) \sigma(m \mid \xi)] d \xi}{\sum_{i \in\{0,1\}} \operatorname{Pr}[\omega=i] \int g(\xi \mid \omega=i, e)[e \sigma(m \mid \omega=i, \xi)+(1-e) \sigma(m \mid \xi)] d \xi}
$$

However, if $m_{1}=\phi$ the manager did not observe $\omega=1$, and thus, $\sigma(m \mid \omega=1, \xi)=0$ for 
any $\xi$. Moreover, no information on $\xi$ is revealed. Therefore, for any $m=\left(\phi, m_{2}\right)$ on the equilibrium path it must be $\int g(\xi \mid \omega=0, e) \sigma(m \mid \omega=0, \xi) d \xi, \int g(\xi \mid \omega=1, e) \sigma(m \mid \xi) d \xi$, and $\int g(\xi \mid \omega=0, e) \sigma(m \mid \xi) d \xi$ obtain the same value. This implies $q(e, m) \equiv \frac{p-e p}{1-e p}$ as required.

Proof of Lemma 2. Expression (A2) below implies $\frac{\partial U}{\partial \lambda}=\frac{1-p}{1-q}\left[\alpha(q, q, \beta)-1_{\{q \geq \beta\}}\right]$ and from $\alpha(q, q, \beta) \in[0,1]$ it follows $\frac{\partial U}{\partial \lambda} \geq 0 \Leftrightarrow q<\beta$. Noting that $\beta=\frac{1}{2}$ concludes the proof.

Proof of Proposition 1. The proof is given for the board's preferences that are stated in Section II. Using $q(e)=\frac{p-e p}{1-e p}$, expression (2) can be rewritten in terms of $q$ as

$$
U(q, \lambda) \equiv \mathbb{E}\left[u_{M}(q, \lambda)\right]=1-\lambda(1-p)\left[\frac{\frac{1}{\lambda}-\alpha(q, q, \beta)-\frac{1-\lambda}{\lambda} \cdot 1_{\{q \geq \beta\}}}{1-q}\right]
$$

where $q \in[\underline{q}, p]$ and $\underline{q}=\frac{p-\bar{e} p}{1-\bar{e} p}$.

An equilibrium exists if $U(q, \lambda)$ has a maximum over $[\underline{q}, p]$. According to Lemma A.1 $\alpha(q, q, \beta)$ is continuous everywhere in $q$. Therefore, if $p<\beta$ then $U(q, \lambda)$ is continuous over $q \in[\underline{q}, p]$ and a maximum exists. If $p \geq \beta$ then $U(q, \lambda)$ is continuous over $[\underline{q}, \beta)$ and $[\max \{\beta, \underline{q}\}, p]$. Therefore, if $U(q, \lambda)$ has a maximum over $[q, \beta)$ it obtains a maximum over $[\underline{q}, p]$ as well. If $U(q, \lambda)$ does not obtain a maximum over $[\underline{q}, \beta)$ then $\sup _{q \in[\underline{q}, \beta)} U(q, \lambda)=$ $\lim _{q \uparrow \beta} U(q, \lambda)$. Note however that $U(\beta, \lambda) \geq \lim _{q \uparrow \beta} U(q, \lambda)$ and therefore the maximum of $U(q, \lambda)$ over $[\underline{q}, p]$ is the maximum over $[\beta, p]$. Overall, $U(q, \lambda)$ has a maximum.

Consider part (i). Suppose $p<\beta$ and for any $p \geq x>y \geq \underline{q}$ let

$$
\Lambda(x, y) \equiv \frac{x-y}{(1-y) \alpha(x, x, \beta)-(1-x) \alpha(y, y, \beta)}
$$

According to Lemma A.1 $\alpha(q, p, \beta)$ increases in its first and second argument. Thus, $\alpha(q, q, \beta)$ increases in $q$ as well. This implies $\Lambda(x, y)>0$ and $U(x, \lambda)>U(y, \lambda) \Leftrightarrow \lambda>\Lambda(x, y)$. Let $q_{\lambda}^{*} \in$ $\arg \max _{q \in[\underline{q}, p]} U(q, \lambda)$. There are three steps for the proof. First, I argue that $\lambda>\lambda^{\prime} \Leftrightarrow q_{\lambda}^{*} \geq q_{\lambda^{\prime}}^{*}$. 
To see why, suppose $\lambda>\lambda^{\prime}$ and by the way of contradiction $q_{\lambda}^{*}<q_{\lambda^{\prime}}^{*}$. Since $q_{\lambda}^{*}$ is a maximizer at $\lambda$ then $\Lambda\left(q_{\lambda^{\prime}}^{*}, q_{\lambda}^{*}\right) \geq \lambda$ and since $q_{\lambda^{\prime}}^{*}$ is a maximizer at $\lambda^{\prime}$ then $\lambda^{\prime} \geq \Lambda\left(q_{\lambda^{\prime}}^{*}, q_{\lambda}^{*}\right)$. Therefore, $\Lambda\left(q_{\lambda^{\prime}}^{*}, q_{\lambda}^{*}\right)>\Lambda\left(q_{\lambda^{\prime}}^{*}, q_{\lambda}^{*}\right)$, a contradiction. Suppose $\lambda<\lambda^{\prime}$ and by the way of contradiction, $q_{\lambda}^{*}>q_{\lambda^{\prime}}^{*}$. Since $q_{\lambda}^{*}$ is a maximizer at $\lambda$ then $\Lambda\left(q_{\lambda^{\prime}}^{*}, q_{\lambda}^{*}\right) \leq \lambda$ and since $q_{\lambda^{\prime}}^{*}$ is a maximizer at $\lambda^{\prime}$ then $\lambda^{\prime} \leq \Lambda\left(q_{\lambda^{\prime}}^{*}, q_{\lambda}^{*}\right)$. Therefore, $\Lambda\left(q_{\lambda^{\prime}}^{*}, q_{\lambda}^{*}\right)<\Lambda\left(q_{\lambda^{\prime}}^{*}, q_{\lambda}^{*}\right)$, a contradiction. Second, note that $q_{0}^{*}=\underline{q}$. Otherwise, if $q_{0}^{*}>\underline{q}$ then the optimality of $q_{0}^{*}$ with respect to $\lambda=0$ implies $\Lambda\left(q_{0}^{*}, \underline{q}\right) \leq 0$. This contradicts the observation that $\Lambda(x, y)>0$ for any $x>y$. Third, note that $q_{1}^{*}=\underline{q}$ if and only if $1 \leq \Lambda(q, \underline{q})$ for all $q \in[\underline{q}, p]$. Given (A3) it is equivalent to the requirement that $q_{\min }=\underline{q}$. This completes part (i).

Consider part (ii) and suppose $p \geq \beta$. According to (A2), conditional on $q \in[\beta, p]$ the optimal choice of $q$ is independent of $\lambda$. I denote this choice by $q^{+}$. Note that $q^{+}$is the unique maximizer at $\lambda$ if and only if $\mathbb{E}\left[u_{M}(q)\right]<\mathbb{E}\left[u_{M}\left(q^{+}\right)\right]$for all $q \in[q, \beta)$. This condition holds if and only if $\lambda<\lambda^{+}$where, ${ }^{24}$

$$
\lambda^{+}(\beta, \underline{q}) \equiv \min _{q \in[\underline{q}, \beta]}\left\{\frac{1}{\alpha(q, q, \beta)+(1-q)\left[\frac{1-\alpha\left(q^{+}, q^{+}, \beta\right)}{1-q^{+}}\right]}\right\}
$$

If $\lambda>\lambda^{+}$then $\arg \max _{q \in[\underline{q}, p]} U(q, \lambda) \subset[\underline{q}, \beta)$ and the analysis is reduced back to the case of $p<\beta$. Note that if $\lambda=\lambda^{+}$then it could be that $q^{+}$is not the unique maximizer. Note that $\lambda^{+}<1$ if and only if there exists $q \in[\underline{q}, \beta)$ such that $\frac{1-\alpha(q, q, \beta)}{1-q}<\frac{1-\alpha\left(q^{+}, q^{+}, \beta\right)}{1-q^{+}}$. Since $q^{+}$is the minimizer of $\frac{1-\alpha(q, q)}{1-q}$ over $[\beta, p]$, then $\lambda^{+}<1 \Leftrightarrow q_{\min }<\beta$. This completes part (ii).

Proof of Corollary 1. Recall $U\left(q_{\lambda}^{*}, \lambda\right)$ is the unconditional probability that the project is approved in equilibrium. Keeping $q_{\lambda}^{*}$ fixed, (A2) implies that $U\left(q_{\lambda}^{*}, \lambda\right)$ increases in $p$. Since the manager maximizes $U(q, \lambda)$ he might choose $q^{\prime} \neq q_{\lambda}^{*}$, but only to the extent that $U\left(q^{\prime}, \lambda\right)>$ $U\left(q_{\lambda}^{*}, \lambda\right)$. Overall, $U\left(q_{\lambda}^{*}, \lambda\right)$ strictly increases in $p$.

\footnotetext{
${ }^{24}$ Implicitly, it is assumed that $\underline{q}(p)<\beta$. Otherwise, the proposition applies for $\lambda^{+}=1$.
} 
If $p<\beta$ then for any $\lambda_{2}>\lambda_{1}$ the optimality of $q_{\lambda_{2}}^{*}$ with respect to $\lambda_{2}$ implies $U\left(q_{\lambda_{2}}^{*}, \lambda_{2}\right) \geq$ $U\left(q_{\lambda_{1}}^{*}, \lambda_{2}\right)$ and Lemma 2 implies $U\left(q_{\lambda_{1}}^{*}, \lambda_{2}\right)>U\left(q_{\lambda_{1}}^{*}, \lambda_{1}\right)$. Therefore, $U\left(q_{\lambda_{2}}^{*}, \lambda_{2}\right)>U\left(q_{\lambda_{1}}^{*}, \lambda_{1}\right)$ as required. Suppose $p \geq \beta$. According to Proposition 1 , if $\lambda<\lambda^{+}$then $q_{\lambda}^{*}=q^{+} \geq \beta$ for all $\lambda$ in this region, where $q^{+}$is independent of $\lambda$. Lemma 2 implies that $U\left(q^{+}, \lambda\right)$ decreases in $\lambda$ in this range. If $\lambda \geq \lambda^{+}$then $q_{\lambda}^{*}<\beta$ and the same reasoning as for the case $p<\beta$ applies.

Proof of Lemma 3. Expression (4) can be rewritten as

$$
V=1-(1-p)\left[\frac{q}{1-q}-\lambda\left(\frac{q}{1-q} \alpha(q, 1, \beta)-\alpha(q, 0, \beta)\right)-\frac{2 q-1}{1-q}(1-\lambda) \cdot 1_{\{q \geq \beta\}}\right]
$$

Suppose $\beta=\frac{1}{2}$. It follows,

$$
\frac{\partial V}{\partial q}=-(1-p)\left[\frac{1-\lambda \alpha(q, 1)-(1-\lambda) \cdot 1_{\{q \geq 1 / 2\}}}{(1-q)^{2}}-\lambda\left(\frac{q}{1-q} \frac{\partial \alpha(q, 1)}{\partial q}-\frac{\partial \alpha(q, 0)}{\partial q}\right)\right]
$$

Since $\frac{\partial \alpha(q, p)}{\partial q}=-\left[p f_{1}\left(s^{*}(q)\right)+(1-p) f_{0}\left(s^{*}(q)\right)\right] \frac{\partial s^{*}(q)}{\partial q}$ for any $p$ and $s^{*}(q) \equiv l^{-1}\left(\frac{1-q}{q}\right)$, it follows that $\frac{q}{1-q} \frac{\partial \alpha(q, 1)}{\partial q}-\frac{\partial \alpha(q, 0)}{\partial q}=0$. Therefore, $\frac{\partial V}{\partial q}=-(1-p)\left[\frac{1-\lambda \alpha(q, 1)-(1-\lambda) \cdot 1_{\{q \geq 1 / 2\}}}{(1-q)^{2}}\right]<0$ as required. Similarly,

$$
\frac{\partial V}{\partial \lambda}=(1-p)\left[\frac{q}{1-q}\left(\alpha(q, 1)-1_{\{q \geq 1 / 2\}}\right)-\left(\alpha(q, 0)-1_{\{q \geq 1 / 2\}}\right)\right]
$$

Since $\frac{f_{1}\left(s^{*}(q)\right)}{f_{0}\left(s^{*}(q)\right)}=\frac{1-q}{q}$ note that

$$
\begin{aligned}
\frac{q}{1-q} \alpha(q, 1)-\alpha(q, 0) & =f_{0}\left(s^{*}(q)\right)\left[\frac{1-F_{1}\left(s^{*}(q)\right)}{f_{1}\left(s^{*}(q)\right)}-\frac{1-F_{0}\left(s^{*}(q)\right)}{f_{0}\left(s^{*}(q)\right)}\right] \\
\frac{q}{1-q}[\alpha(q, 1)-1]-[\alpha(q, 0)-1] & =f_{0}\left(s^{*}(q)\right)\left[\frac{F_{0}\left(s^{*}(q)\right)}{f_{0}\left(s^{*}(q)\right)}-\frac{F_{1}\left(s^{*}(q)\right)}{f_{1}\left(s^{*}(q)\right)}\right]
\end{aligned}
$$

The MLRP implies $\frac{f_{1}(s)}{1-F_{1}(s)}<\frac{f_{0}(s)}{1-F_{0}(s)}$ and $\frac{F_{0}\left(s^{*}(q)\right)}{f_{0}\left(s^{*}(q)\right)}>\frac{F_{1}\left(s^{*}(q)\right)}{f_{1}\left(s^{*}(q)\right)}$ for all $s$. Thus, the r.h.s of both expressions is strictly positive and $\frac{\partial V}{\partial \lambda}>0$ as required. 
Proof of Proposition 2. Suppose $p<\frac{1}{2}$. According to Proposition $1 q_{0}^{*}=q(\bar{e})$ and by definition $q_{1}^{*}=q_{\min }$. Thus, according to (A5)

$$
\begin{aligned}
V\left(q_{0}^{*}, 0\right) & =1-(1-p) \frac{q(\bar{e})}{1-q(\bar{e})} \\
V\left(q_{1}^{*}, 1\right) & =1-(1-p)\left[\frac{q_{\min }}{1-q_{\min }}\left(1-\alpha\left(q_{\min }, 1\right)\right)+\alpha\left(q_{\min }, 0\right)\right]
\end{aligned}
$$

If $\alpha(q, q) \leq q$ for all $q \in(0, p]$ then $q_{\min }=q(\bar{e})=0$ when $\bar{e}=1$. According to Proposition $1 q_{\lambda}^{*}$ increases in $\lambda$. Thus, $q_{\lambda}^{*}=0$ for all $\lambda \in[0,1]$. Therefore, $V\left(q_{\lambda}^{*}, \lambda\right)=1$ for all $\lambda$ and $\lambda^{*}=1$ by definition. Suppose instead that there is $\chi \in(0, p]$ such that $\alpha(\chi, \chi)>\chi$. Let

$q_{\text {global }} \in \arg \min _{q \in[0, p]}\left\{\frac{1-\alpha(q, q)}{1-q}\right\}$ and note that $q_{\text {global }}>0$. Consider all $\bar{e}$ such that $q(\bar{e})<$ $q_{\text {global }}$. In this region $q_{\text {min }}=q_{g l o b a l}$ and hence $V\left(q_{1}^{*}, 1\right)<V\left(q_{0}^{*}, 0\right)$ if and only if $\frac{q(\bar{e})}{1-q(\bar{e})}<$ $\frac{q_{\text {global }}}{1-q_{\text {global }}}\left(1-\alpha\left(q_{\text {global }}, 1\right)\right)+\alpha\left(q_{\text {global }}, 0\right)$. Note that the r.h.s is independent of $\bar{e}$ and the l.h.s equals $\frac{p}{1-p}(1-\bar{e})$. Therefore, there is $\underline{e}<1$ independent of $\lambda$ such that $V\left(q_{1}^{*}, 1\right)<V\left(q_{0}^{*}, 0\right) \Leftrightarrow$ $\bar{e} \in[\underline{e}, 1]$. This implies $\lambda^{*}<1$ as required.

Suppose $p \geq \frac{1}{2}$. Proposition 1 implies $q_{\lambda}^{*}=q^{+}$for all $\lambda \in\left[0, \lambda^{+}\right]$where $\lambda^{+}>0$. From Lemma 3 it follows that $V\left(q^{+}, \lambda^{+}\right)>V\left(q^{+}, 0\right)$, implying $\lambda^{*} \geq \lambda^{+}>0$.

\section{B Proofs of Section II}

Remark: For any $(\bar{e}, \lambda, \beta)$ let $q_{\bar{e}, \lambda, \beta}^{*}$ be the equilibrium level of $q(e)$ and $V(q, \lambda, \beta)$ be shareholders' expected value for a given $q$. The proof of Proposition 3 follows two auxiliary lemmas.

Lemma A.2 For any $\lambda \in(0,1]$ and $\bar{e}, \lim _{\beta \rightarrow 1} q_{\bar{e}, \lambda, \beta}^{*}=\underline{q}$ and $\lim _{\beta \rightarrow 1} V\left(q_{\bar{e}, \lambda, \beta}^{*}, \lambda, \beta\right)=p \bar{e}+$ $(1-p)$

Proof. Suppose on the contrary $\lim _{\beta \rightarrow 1} q_{\bar{e}, \lambda, \beta}^{*}>\underline{q}$. Then, there exists $\left\{\beta_{n}\right\}_{n=1}^{\infty}$ such that 
$\lim _{n \rightarrow \infty} \beta_{n}=1, q_{\bar{e}, \lambda, \beta_{n}}^{*}>\underline{q}$, and $\beta_{n}>p$ for all $n$. The optimality of $q_{\bar{e}, \lambda, \beta_{n}}^{*} \operatorname{implies} U\left(\underline{q}, \lambda, \beta_{n}\right) \leq$ $U\left(q_{\bar{e}, \lambda, \beta_{n}}^{*}, \lambda, \beta_{n}\right)$. Since $\beta_{n}>p \geq q_{\bar{e}, \lambda, \beta_{n}}^{*}>\underline{q}$, it follows that $\Lambda\left(q_{\bar{e}, \lambda, \beta_{n}}^{*}, \underline{q}, \beta_{n}\right) \leq \lambda$ for all $n$. Lemma A.1 implies that $\alpha\left(q, q, \beta_{n}\right)<\alpha\left(p, p, \beta_{n}\right)$ for all $q$. Thus, it follows from (A3) that $\frac{1}{\alpha\left(p, p, \beta_{n}\right)\left(\frac{1-q}{q_{\tilde{e}, \lambda, \beta_{n}-\underline{q}}^{*}}\right)+\alpha\left(\underline{q}, \underline{q}, \beta_{n}\right)}<\Lambda\left(q_{\bar{e}, \lambda, \beta_{n}}^{*}, \underline{q}, \beta_{n}\right)$. Since $\lim _{n \rightarrow \infty} q_{\bar{e}, \lambda, \beta_{n}}^{*}>\underline{q}$ and $\lim _{n \rightarrow \infty} \alpha\left(q, q, \beta_{n}\right)=$ 0 for all $q \in[\underline{q}, p]$ then $\lim _{n \rightarrow \infty} \Lambda\left(q_{\bar{e}, \lambda, \beta_{n}}^{*}, \underline{q}, \beta_{n}\right)=\infty$, contradicting $\Lambda\left(q_{\bar{e}, \lambda, \beta_{n}}^{*}, \underline{q}, \beta_{n}\right) \leq \lambda$. Since $\lim _{\beta \rightarrow 1} q_{\bar{e}, \lambda, \beta}^{*}=\underline{q}$ and $\lim _{\beta \rightarrow 1} \alpha(q, p, \beta)=0$, according to (A5), $\lim _{\beta \rightarrow 1} V\left(q_{\bar{e}, \lambda, \beta}^{*}, \lambda, \beta\right)=$ $1-(1-p) \frac{\underline{q}}{1-\underline{q}}=p \bar{e}+(1-p)$ as required.

Lemma A.3 Suppose there exists $q_{0} \in(0, p]$ such that $U\left(q_{0}, \lambda, \frac{1}{2}\right)>U\left(0, \lambda, \frac{1}{2}\right)$ for $\lambda>0$. Then, there exists $e_{\lambda}<1$ such that if $\bar{e} \in\left[e_{\lambda}, 1\right]$ then $\beta^{*}>\frac{1}{2}$.

Proof. Suppose on the contrary that for some $\lambda>0$ there exist $q_{0} \in(0, p]$ such that $U\left(q_{0}, \lambda, \frac{1}{2}\right)>U\left(0, \lambda, \frac{1}{2}\right)$ and $\left\{\bar{e}_{n}\right\}_{n=1}^{\infty}$ where $\lim _{n \rightarrow \infty} \bar{e}_{n}=1, \bar{e}_{n}<1$ and $\beta_{n}^{*} \leq \frac{1}{2}$ for all $n$. Also, let $\beta_{\lim }^{*} \equiv \lim _{n \rightarrow \infty} \beta_{n}^{*} \in\left[0, \frac{1}{2}\right]$ and $\alpha_{\lim }^{*} \equiv \lim _{n \rightarrow \infty} \alpha\left(q_{\bar{e}_{n}, \lambda, \beta_{n}^{*}}^{*}, q_{\bar{e}_{n}, \lambda, \beta_{n}^{*}}^{*}, \beta_{n}^{*}\right) \in[0,1]$.

I start by arguing that $\lim _{n \rightarrow \infty} q_{\bar{e}_{n}, \lambda, \beta_{n}^{*}}^{*}=0$. To see why, note that the optimality of $\beta_{n}^{*}$ implies $V\left(q_{\bar{e}_{n}, \lambda, \beta_{n}^{*}}^{*}, \lambda, \beta_{n}^{*}\right) \geq V\left(q_{\bar{e}_{n}, \lambda, \beta}^{*}, \lambda, \beta\right)$ for all $n$ and $\beta>\frac{1}{2}$. Lemma A.2 implies $\lim _{\beta \rightarrow 1} V\left(q_{\bar{e}_{n}, \lambda, \beta}^{*}, \lambda, \beta\right)=p \bar{e}_{n}+(1-p)$. Let $\left\{\beta_{n}^{\prime}\right\}$ such that $\lim _{n \rightarrow \infty} \beta_{n}^{\prime}=1$. Then, $V\left(q_{\bar{e}_{n}, \lambda, \beta_{n}^{*}}^{*}, \lambda, \beta_{n}^{*}\right) \geq$ $V\left(q_{\bar{e}_{n}, \lambda, \beta}^{*}, \lambda, \beta_{n}^{\prime}\right)$ for large $n$ and $\lim _{n \rightarrow \infty} V\left(q_{\bar{e}_{n}, \lambda, \beta_{n}^{*}}^{*}, \lambda, \beta_{n}^{*}\right)=1$. This implies $\lim _{n \rightarrow \infty} q_{\bar{e}_{n}, \lambda, \beta_{n}^{*}}^{*}=0$ as required.

Next, consider two cases. First, suppose $\alpha_{\lim }^{*}=0$. Since $\beta_{\lim }^{*} \leq \frac{1}{2}$, the definitions of $\alpha(q, q, \beta)$ and $s^{*}(q, \beta)$ imply that $\lim _{n \rightarrow \infty} \frac{\beta_{n}^{*}}{1-\beta_{n}^{*}} \frac{1-q_{\tilde{e}_{n}, \lambda, \beta_{n}^{*}}^{*}}{q_{\bar{e}_{n}, \lambda, \beta_{n}^{*}}^{*}}=\infty$. Since $\lim _{n \rightarrow \infty} q_{\bar{e}_{n}, \lambda, \beta_{n}^{*}}^{*}=0$ then $q_{\bar{e}_{n}, \lambda, \beta_{n}^{*}}^{*}$ converges to zero faster than $\beta_{n}^{*}$. Thus, $\min \left\{\beta_{n}^{*}, q_{0}\right\}>q_{\bar{e}_{n}, \lambda, \beta_{n}^{*}}^{*}$ for sufficiently large $n$. The optimality of $q_{\bar{e}_{n}, \lambda, \beta_{n}^{*}}^{*}$ implies $U\left(q_{0}, \lambda, \beta_{n}^{*}\right) \leq U\left(q_{\bar{e}_{n}, \lambda, \beta_{n}^{*}}^{*}, \lambda, \beta_{n}^{*}\right)$ for sufficiently large $n$. Thus, in the limit, $\alpha\left(q_{0}, q_{0}, \beta_{\lim }^{*}\right)+\frac{1-\lambda}{\lambda} \cdot 1_{\left\{q_{0} \geq \beta_{\mathrm{lim}}^{*}\right\}} \leq \frac{q_{0}}{\lambda}$. However, the assumption that $U\left(q_{0}, \lambda, \frac{1}{2}\right)>U\left(0, \lambda, \frac{1}{2}\right)$ implies $\frac{q_{0}}{\lambda}<\alpha\left(q_{0}, q_{0}, \frac{1}{2}\right)+\frac{1-\lambda}{\lambda} \cdot 1_{\left\{q_{0} \geq \frac{1}{2}\right\}}$. Since $\beta_{\lim }^{*} \leq \frac{1}{2}$ and $\alpha(q, q, \beta)$ strictly decreases in $\beta$ we get a contradiction. 
Second, suppose $\alpha_{\lim }^{*}>0$. Since $\lim _{n \rightarrow \infty} q_{\bar{e}_{n}, \lambda, \beta_{n}^{*}}^{*}=0$, the definitions of $\alpha(q, q, \beta)$ and $s^{*}(q, \beta)$ imply $\beta_{\lim }^{*}=0$. Note that $\alpha_{\lim }^{*}<1$ since if $\alpha_{\lim }^{*}=1$ then the probably of approval conditional on non-disclosure converges to one. This implies $\lim _{n \rightarrow \infty} V\left(q_{\bar{e}_{n}, \lambda, \beta_{n}^{*}}^{*}, \lambda, \beta_{n}^{*}\right)=p$. However, in this case and according to (A5), as long as $\lambda>0$ shareholders get strictly more than $p$ for any $q$ if they set $\beta=\frac{1}{2}$. Therefore, $\alpha_{\lim }^{*}<1$. The optimality of $q_{\bar{e}_{n}, \lambda, \beta_{n}^{*}}^{*}$ implies that $U\left(q_{0}, \lambda, \beta_{n}^{*}\right) \leq U\left(q_{\bar{e}_{n}, \lambda, \beta_{n}^{*}}^{*}, \lambda, \beta_{n}^{*}\right)$ for large $n$. Since $\beta_{\lim }^{*}=0=\lim _{n \rightarrow \infty} q_{\bar{e}_{n}, \lambda, \beta_{n}^{*}}$ the limit of $U\left(q_{0}, \lambda, \beta_{n}^{*}\right) \leq U\left(q_{\bar{e}_{n}, \lambda, \beta_{n}^{*}}^{*}, \lambda, \beta_{n}^{*}\right)$ implies $\alpha_{\lim }^{*} \geq \frac{1}{\lambda}-\frac{1-\lambda}{\lambda} \cdot 1\left\{\lim _{n \rightarrow \infty} \frac{q_{\bar{e}_{n}, \lambda, \beta_{n}^{*}}^{*}}{\beta_{n}^{*}} \geq 1\right\} \geq 1$, a contradiction.

Proof of Proposition 3. Suppose $\lambda=0$ and note that based on (A2) and (A5) $U=$ $1-\frac{1-p}{1-q} \cdot 1_{\{q<\beta\}}$ and $V=1-(1-p) \frac{q-(2 q-1) \cdot 1_{\{q \geq \beta\}}}{1-q}$. It follows that $q^{*}=\underline{q}$ if $\beta>p$ and otherwise $q^{*} \in[\beta, p]$. Let $e_{\lambda=0}=\max \left\{\frac{2 p-1}{p}, 0\right\}$ and note that $e_{0}<1$. If $p<\frac{1}{2}$ then $\beta=\frac{1}{2}$ implies $q^{*}=\underline{q}$ and hence $\beta^{*}=\frac{1}{2}$ for all $\bar{e}$. Suppose $p>\frac{1}{2}$ and $\bar{e}>e_{\lambda=0}$. If $\beta \leq p$ then $q^{*} \geq \beta$ and $V\left(q^{*}\right)=p$. If $\beta>p$ then $q^{*}=\underline{q}$ and $V\left(q^{*}\right)=1-(1-p) \frac{\underline{q}}{1-\underline{q}}=1-p+\bar{e} p$. Thus, $\beta^{*}>\frac{1}{2}$ if and only if $1-p+\bar{e} p>p \Leftrightarrow \bar{e}>e_{\lambda=0}$. This completes the proof for $\lambda=0$.

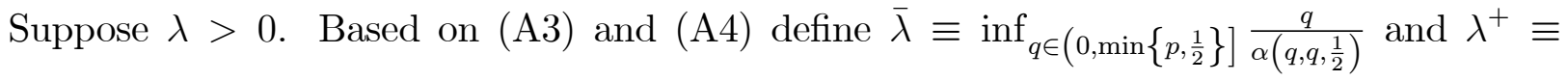
$\lambda^{+}\left(\frac{1}{2}, 0\right)$. Note that $\lambda^{+}>0$. I start by proving that if $p<\frac{1}{2}$ and $\lambda \leq \bar{\lambda}$, or $p \geq \frac{1}{2}$ and $\lambda \in\left[\lambda^{+}, \bar{\lambda}\right]$, then $q_{\bar{e}=1, \lambda, \beta=\frac{1}{2}}^{*}=0$. First, if $p<\frac{1}{2}$ then $U\left(q, 0 ; \beta=\frac{1}{2}\right)<U\left(0,0 ; \beta=\frac{1}{2}\right)$ for all $q \in(0, p]$. Second, if $0<\lambda \leq \bar{\lambda}$ then $U\left(q, \lambda ; \beta=\frac{1}{2}\right)<U\left(0, \lambda ; \beta=\frac{1}{2}\right)$ for any $q \in$ $\left(0, \min \left\{p, \frac{1}{2}\right\}\right]$. Third, if $\lambda \geq \lambda^{+}$then by definition of $\lambda^{+}$there is $q \in\left[0, \frac{1}{2}\right)$ such that $U\left(q^{+}, \lambda ; \beta=\frac{1}{2}\right)<U\left(q, \lambda ; \beta=\frac{1}{2}\right)$. Combined, the three cases establish the argument.

Next, I show that if $p<\frac{1}{2}$ and $\lambda \leq \bar{\lambda}$, or $p \geq \frac{1}{2}$ and $\lambda \in\left[\lambda^{+}, \bar{\lambda}\right]$, there is $e_{\lambda}<1$ such that if $\bar{e} \in\left[e_{\lambda}, 1\right]$ then $q_{\bar{e}=1, \lambda, \beta=\frac{1}{2}}^{*}=q(\bar{e})$. To see why, note that previous argument established that the global maximum of $U\left(q, \lambda, \frac{1}{2}\right)$ over $[0, p]$ is obtained at $q=0$. Since $U\left(q, \lambda, \frac{1}{2}\right)$ is continuous in $q$ at the neighborhood of zero, for small $\varepsilon>0$, the global maximum of $U\left(q, \lambda, \frac{1}{2}\right)$ over $[\varepsilon, p]$ is obtained at $q=\varepsilon$. Let $e_{\lambda}$ be such that $q\left(e_{\lambda}\right)=\varepsilon$. Then for any $\bar{e} \in\left[e_{\lambda}, 1\right]$ the 
global maximum of $U\left(q, \lambda, \frac{1}{2}\right)$ over $[q(\bar{e}), p]$ is obtained at $q=q(\bar{e})$ as required. We conclude that when the board is unbiased, under the conditions above, the managerial learning intensity is at its highest level and therefore shareholders cannot benefit from a biased board, that is, $\beta^{*}=\frac{1}{2}$.

If $\lambda>\bar{\lambda}$ there is $q_{0} \in\left(0, \min \left\{p, \frac{1}{2}\right\}\right]$ such that $\lambda>\Lambda\left(q, 0, \frac{1}{2}\right)$. If $p \geq \frac{1}{2}$ and $\lambda<\lambda^{+}$ there is $q_{0} \in\left[\frac{1}{2}, p\right]$ such that $1>\Lambda\left(q_{0}, 0, \frac{1}{2}\right)$. Either way, there exists $q_{0} \in(0, p]$ such that $U\left(q_{0}, \lambda, \frac{1}{2}\right)>U\left(0, \lambda, \frac{1}{2}\right)$ and Lemma A.3 establishes the result.

\section{Proofs of Section III}

Remark: The proofs of this section rely on Lemma A.4 which provides implicit conditions for the existence of equilibria and characterizes them.

\section{Lemma A.4}

(i) There is an equilibrium in which $q^{*}=0$ if and only if $p \leq \frac{1}{2}$ and $\lambda \leq \frac{1}{2 \pi}$. In this equilibrium, $\Delta^{*}=0$ and conditional on non-disclosure, the board rejects the project with probability one.

(ii) There is an equilibrium in which $q^{*}=\frac{1}{1+\frac{\pi}{1-\pi} \frac{1-\Delta^{*}}{1+\Delta^{*}}}$ if and only if either

$$
\max \left\{\frac{1-\lambda \pi(3-2 \pi)}{(1-\lambda \pi)(2 \pi-1)}, \frac{p-\pi}{p(1-\pi)+\pi(1-p)}\right\}<\Delta^{*}<\min \{0,2 \lambda \pi-1,2 p-1\}
$$

or

$$
2 p-1<\Delta^{*}<\min \{0,2 \lambda \pi-1\}
$$


where $\Delta^{*}$ satisfies

$$
\Delta^{*} \equiv \Gamma\left(\frac{1}{1+\frac{1-\lambda}{\lambda} \frac{1}{q^{*}(1-\pi)+\left(1-q^{*}\right) \pi}}\right)-\Gamma\left(\frac{1}{1+\frac{1-\lambda}{\lambda} \frac{\frac{p-q^{*}}{1-p}}{\frac{p-q^{*}}{1-p}+\left[q^{*} \pi+\left(1-q^{*}\right)(1-\pi)\right]}}\right)
$$

In this equilibrium, conditional on non-disclosure, the board approves the project the if and only if $s=1$.

(iii) There is an equilibrium in which $q^{*}=\frac{1+\Delta^{*}}{2}$ if and only if

$$
0<\Delta^{*} \leq \min \left\{\frac{1-\lambda}{1+\lambda(1-2 \pi)}, \frac{1-\lambda \pi(3-2 \pi)}{(1-\lambda \pi)(2 \pi-1)}, 2 p-1\right\}
$$

where $\Delta^{*}$ satisfies

$$
\Delta^{*}=\Gamma(1)-\Gamma\left(\frac{1}{1+\frac{1-\lambda}{\lambda} \frac{\frac{p-q^{*}}{1-p}+1}{\frac{p-q^{*}}{1-p}+\left[q^{*} \pi+\left(1-q^{*}\right)(1-\pi)\right]}}\right)
$$

In this equilibrium, conditional on non-disclosure, the board rejects the project if and only if $s=0$.

(iv) There is no other equilibrium.

Proof. Shareholders' beliefs depend only on $a$ but not on $e$ or $m$, and hence from the manager and the board's point of view, they are fixed. Let $\Delta^{*}$ be the equilibrium value of $\Gamma(\operatorname{Pr}[\rho=1 \mid a=0])-\Gamma(\operatorname{Pr}[\rho=1 \mid a=1])$ and not that $\Gamma(1)<1$ guarantees that $\Delta^{*} \in(-1,1)$.

I start by noting that unless $q=p$ then in equilibrium the board (both the layman and the expert) plays a pure strategy. Otherwise, the manager will always find it optimal to marginally increase $q$ and thereby discretely increase the probably that the project is approved.

Suppose the manager chooses $q$ and does not disclose the information. Recall that conditional on non-disclosure the layman board approves the project if and only if $q \geq \frac{1+\Delta^{*}}{2}$. The 
expert board with signal $s \in\{0,1\}$ approves the project if and only if $q>\frac{1}{1+\frac{\pi}{1-\pi} \frac{1-\Delta^{*}}{1+\Delta^{*}}}$ when $s=1$ and if and only if $q>\frac{1}{1+\frac{1-\pi}{\pi} \frac{1-\Delta^{*}}{1+\Delta^{*}}}$ when $s=0$. Thus, the probability the board approves the project conditional on non-disclosure is

$$
\alpha\left(q, p, \Delta^{*}\right)= \begin{cases}0 & \text { if } q<\frac{1}{1+\frac{\pi}{1-\pi} \frac{1-\Delta^{*}}{1+\Delta^{*}}} \\ p \pi+(1-p)(1-\pi) & \text { if } q \in\left[\frac{1}{\left.1+\frac{\pi}{1-\pi} \frac{1-\Delta^{*}}{1+\Delta^{*}}, \frac{1}{1+\frac{1-\pi}{\pi} \frac{1-\Delta^{*}}{1+\Delta^{*}}}\right)}\right. \\ 1 & \text { if } q \geq \frac{1}{1+\frac{1-\pi}{\pi} \frac{1-\Delta^{*}}{1+\Delta^{*}}}\end{cases}
$$

The manager's expected utility is given by

$$
\mathbb{E}\left[u_{M}\left(q, \Delta^{*}\right)\right]=1-\lambda(1-p)\left[\frac{\frac{1}{\lambda}-\alpha\left(q, q, \Delta^{*}\right)-\frac{1-\lambda}{\lambda} \cdot 1_{\left\{q \geq \frac{1+\Delta^{*}}{2}\right\}}}{1-q}\right]
$$

From (A13) and (A14) it follows that the manager optimal decision of $q$ is either zero, min $\left\{\frac{1}{1+\frac{\pi}{1-\pi} \frac{1-\Delta^{*}}{1+\Delta^{*}}}, p\right\}$ $\min \left\{\frac{1+\Delta^{*}}{2}, p\right\}$, or $\min \left\{\frac{1}{1+\frac{1-\pi}{\pi} \frac{1-\Delta^{*}}{1+\Delta^{*}}}, p\right\}$.

According to Lemma A.9 in no equilibrium the project is approved with probability one. Therefore, $q^{*}=\frac{1}{1+\frac{1-\pi}{\pi} \frac{1-\Delta^{*}}{1+\Delta^{*}}}$ is never an equilibrium. This establishes part (iv) of the Lemma. Moreover, if $\frac{1}{1+\frac{1-\pi}{\pi} \frac{1-\Delta^{*}}{1+\Delta^{*}}} \leq p$ the manager has strict incentives to deviate to $q=\frac{1}{1+\frac{1-\pi}{\pi} \frac{1-\Delta^{*}}{1+\Delta^{*}}}$ and obtain the highest welfare possible, one. Therefore, I must require that in any equilibrium $\frac{1}{1+\frac{1-\pi}{\pi} \frac{1-\Delta^{*}}{1+\Delta^{*}}}>p$ which holds if and only if

$$
\Delta^{*}>\frac{p-\pi}{p(1-\pi)+\pi(1-p)}
$$

According to $(\mathrm{A} 14)$ and for any $\Delta^{*} \in(-1,1)$ it follows that $\mathbb{E}\left[u_{M}\left(0, \Delta^{*}\right)\right]>\mathbb{E}\left[u_{M}\left(\frac{1+\Delta^{*}}{2}, \Delta^{*}\right)\right]$ if and only if

$$
\Delta^{*}>\frac{1-\lambda}{1+\lambda(1-2 \pi)}
$$


$\mathbb{E}\left[u_{M}\left(0, \Delta^{*}\right)\right]>\mathbb{E}\left[u_{M}\left(\frac{1}{1+\frac{\pi}{1-\pi} \frac{1-\Delta^{*}}{1+\Delta^{*}}}, \Delta^{*}\right)\right]$ if and only if

$$
\begin{gathered}
\Delta^{*}>2 \lambda \pi-1, \\
\mathbb{E}\left[u_{M}\left(\frac{1+\Delta^{*}}{2}, \Delta^{*}\right)\right]>\mathbb{E}\left[u_{M}\left(\frac{1}{1+\frac{\pi}{1-\pi} \frac{1-\Delta^{*}}{1+\Delta^{*}}}, \Delta^{*}\right)\right] \text { if and only if } \\
\Delta^{*}<\frac{1-\lambda \pi(3-2 \pi)}{(1-\lambda \pi)(2 \pi-1)} .
\end{gathered}
$$

Consider an equilibrium in which $q^{*}=0$. In this equilibrium, regardless of its expertise, the board approves the project conditional on disclosure and rejects the project conditional on non-disclosure and, therefore, $\Delta^{*}=0$. Condition (A15) does not bind when $\Delta^{*}=0$. Condition (A16) implies that the option $q>\frac{1}{2}$, if feasible, always dominates $q=0$. Hence, this equilibrium exits only if $p \leq \frac{1}{2}$ (if $q=p=\frac{1}{2}$ the layman board is indifferent and hence I assume that he would reject the project). When $p<\frac{1}{2}$, condition (A17) requires that $2 \lambda \pi-1<0$. I conclude, an equilibrium with $q^{*}=0$ exists if and only if $p<\frac{1}{2}$ and $2 \lambda \pi-1<0$. This establishes part (i).

Consider an equilibrium in which $q^{*}=\frac{1}{1+\frac{\pi}{1-\pi} \frac{1-\Delta^{*}}{1+\Delta^{*}}}$. In this equilibrium, the layman board rejects the project conditional on non-disclosure and hence $\Delta^{*}<0$. The option $q^{*}=\frac{1}{1+\frac{\pi}{1-\pi} \frac{1-\Delta^{*}}{1+\Delta^{*}}}$ is feasible if and only if $p \geq \frac{1}{1+\frac{\pi}{1-\pi} \frac{1-\Delta^{*}}{1+\Delta^{*}}}$. Since $p>1-\pi$ this condition never binds given the requirement that $\Delta^{*}<0$. Condition (A15) and the reversed of condition (A17) imply that it is necessary that

$$
\frac{p-\pi}{p(1-\pi)+\pi(1-p)}<\Delta^{*}<\min \{0,2 \lambda \pi-1\}
$$

If $\frac{1+\Delta^{*}}{2} \leq p$ then I also need to require the reversed of condition (A18). This yields condition (A8). If $\frac{1+\Delta^{*}}{2}>p$ then the option $q=\frac{1+\Delta^{*}}{2}$ is not feasible and noting that $2 p-1>\frac{p-\pi}{p(1-\pi)+\pi(1-p)}$ yields condition (A9). Moreover, in order to be consistent with shareholders' expectation, $\Delta^{*}$ must be the fixed point of (A10). Note that the r.h.s of (A10) decreases in $\Delta^{*}$, it is strictly 
negative, and bounded away from below from minus one. Therefore, there exists a unique solution for (A10). This establishes part (ii).

Consider an equilibrium in which $q^{*}=\frac{1+\Delta^{*}}{2}$. In this equilibrium, the layman board approves the projects with probability one and hence $\Delta^{*}>0$. If $\Delta^{*}>0$ then (A15) does not bind. The option $q=\frac{1+\Delta^{*}}{2}$ is feasible if and only if $p \geq \frac{1+\Delta^{*}}{2}$. Combining condition (A18) and the reversed of condition (A16) yields condition (A11). Moreover, in order to be consistent with shareholders' expectation, $\Delta^{*}$ must be the fixed point of (A12). Note that the r.h.s of (A12) is increasing in $\Delta^{*}$, strictly positive, and it is bounded away from above from 1 . Therefore, there exists a solution to (A12). This establishes part (iii).

Proof of Lemma 4. There are several cases to consider. First, suppose $p \leq \frac{1}{2}$ and $\lambda<\frac{1}{2 \pi}$, and let $\bar{\Gamma} \equiv 1$. Part (i) of Lemma A.4 guarantees that if $\Gamma(1) \leq \bar{\Gamma}$ then an equilibrium in which $q^{*}=0$ exists. Second, suppose $p<\frac{1}{2}$ and $\frac{1}{2 \pi}<\lambda<\frac{1}{\pi(3-2 \pi)}$, and let $\bar{\Gamma} \equiv 1-2 p$. If $\Gamma(1)<\bar{\Gamma}$ the a solution for (A10) must be strictly negative and greater than $-\bar{\Gamma}$ and hence satisfies condition (A9). Part (ii) of Lemma A.4 guarantees that an equilibrium in which $q^{*}=\frac{1}{1+\frac{\pi}{1-\pi} \frac{1-\Delta^{*}}{1+\Delta^{*}}}$ exists. Third, suppose $p>\frac{1}{2}$ and $\lambda<\frac{1}{\pi(3-2 \pi)}$, and let $\bar{\Gamma} \equiv \min \left\{\frac{1-\lambda}{1+\lambda(1-2 \pi)}, \frac{1-\lambda \pi(3-2 \pi)}{(1-\lambda \pi)(2 \pi-1)}, 2 p-1\right\}$. Note that $\bar{\Gamma}>0$. If $\Gamma(1)<\bar{\Gamma}$ the a solution for (A12) must be strictly positive and smaller than $\bar{\Gamma}$ and hence satisfies condition (A11). Therefore, Part (iii) of Lemma A.4 guarantees that an equilibrium in which $q^{*}=\frac{1+\Delta^{*}}{2}$ exists. Finally suppose $\lambda \geq \frac{1}{\pi(3-2 \pi)}$ and let $\bar{\Gamma} \equiv$ $-\max \left\{\frac{1-\lambda \pi(3-2 \pi)}{(1-\lambda \pi)(2 \pi-1)}, \frac{p-\pi}{p(1-\pi)+\pi(1-p)}\right\}$. Note that $\bar{\Gamma}>0$. If $\Gamma(1)<\bar{\Gamma}$ the a solution for (A10) must be strictly negative and greater than $-\bar{\Gamma}$. This implies that either (A8) (if $\left.p<\frac{1}{2}\right)$ or $($ A9) (if $p \geq \frac{1}{2}$ ) hold. Part (ii) of Lemma A.4 guarantees that an equilibrium in which $q^{*}=\frac{1}{1+\frac{\pi}{1-\pi} \frac{1-\Delta^{*}}{1+\Delta^{*}}}$ exists.

Proof of Lemma 5. Suppose in equilibrium $\Delta^{*} \leq 0$, Lemma A.4 implies that either $q^{*}=0$ or $q^{*}=\frac{1}{1+\frac{\pi}{1-\pi} \frac{1-\Delta^{*}}{1+\Delta^{*}}}$. In the latter case, $q^{*} \leq \frac{1}{2}$ if and only if $\Delta^{*} \leq 2 \pi-1$. Since $\Delta^{*} \leq 0$ and 
$\pi>\frac{1}{2}$ then $q^{*}<\frac{1}{2}$. Suppose in equilibrium $\Delta^{*}>0$. Lemma A.4 implies that either $q^{*}=\frac{1+\Delta^{*}}{2}$ or $q^{*}=\frac{1}{1+\frac{\pi}{1-\pi} \frac{1-\Delta^{*}}{1+\Delta^{*}}}$. In the former case, $\Delta^{*}>0 \Rightarrow q^{*}>\frac{1}{2}$. In the latter case, part (ii) of the Lemma A.4 requires $\Delta^{*}>2 p-1$. Since $\pi>p$ then in such equilibrium it is necessary that $\Delta^{*}>2 \pi-1$ as well. As was argued above, this implies $q^{*}>\frac{1}{2}$. Either way, $\Delta^{*}>0$ if and only if $q^{*}>\frac{1}{2}$.

Proof of Proposition 4. I start by proving the statement on the likelihood of the project's approval. I compare the unconditional probability that the project is approved in equilibria when $\Gamma$ satisfies the assumptions of Section III, with equilibria in which $\Gamma \equiv 0$ and hence there are no reputation concerns. In that case, conditions (A10) and (A12) in Lemma A.4 are not required as $\Delta$ is determined independently of shareholders' expectations of the board's behavior and is fixed exogenously to be zero. Therefore, for most parts (ignoring the strict inequalities in conditions (A8), (A9), and (A11)), Lemma A.4 also includes the case of $\Delta \equiv 0$.

There are several cases to consider. First, suppose $p<\frac{1}{2}$ and $\lambda<\frac{1}{2 \pi}$. Conditions (A8) and (A11) are violated for any $\Delta$. Thus, if $\Delta \equiv 0$ then Lemma A.4 implies $q^{*}=0$ is the only equilibrium. With reputation concerns, Lemma A.4 implies that either $q^{*}=0$ or $q^{*}=\frac{1}{1+\frac{\pi}{1-\pi} \frac{1-\Delta^{*}}{1+\Delta^{*}}}$ where (A9) must hold (that is, $\Delta^{*}<0$ ). If $q^{*}=0$ then the manager's expected utility is $p$ whether or not the board has reputation concerns. If $q^{*}=\frac{1}{1+\frac{\pi}{1-\pi} \frac{1-\Delta^{*}}{1+\Delta^{*}}}$ then according to (A17) the manager's expected utility is strictly greater than $p$ if and only if $\Delta^{*}<2 \lambda \pi-1$. Condition (A9) implies that $\Delta^{*}<2 \lambda \pi-1$ and hence in this region the manager weakly gains power.

Second, suppose $p<\frac{1}{2}$ and $\lambda \geq \frac{1}{2 \pi}$ or $p \geq \frac{1}{2}$ and $\lambda \geq \frac{1}{\pi(3-2 \pi)}$. Condition (A11) is violated for any $\Delta$. If $\Delta \equiv 0$ then conditions (A9) or (A8) are satisfied and hence in the absence of reputation concerns the manager chooses $q^{*}=1-\pi$. With reputation concerns, Lemma A.4 implies that necessarily $q^{*}=\frac{1}{1+\frac{\pi}{1-\pi} \frac{1-\Delta^{*}}{1+\Delta^{*}}}<1-\pi$ where $\Delta^{*}<0$. In both scenarios and conditional on non-disclosure the board approves the project if and only if $s=1$. However, in the former case, the manager collects less information ( $q^{*}$ is higher) and hence the probability 
of disclosing $\omega=1$ is strictly lower. Therefore, the overall probability of project's approval is higher when the board has reputation concerns.

Last, suppose $p \geq \frac{1}{2}$ and $\lambda<\frac{1}{\pi(3-2 \pi)}$. Conditions (A8) and (A9) are violated for any $\Delta$. If $\Delta \equiv 0$ then condition (A11) is satisfied and hence in the absence of reputation concerns the manager chooses $q^{*}=\frac{1}{2}$. With reputation concerns, Lemma A.4 implies that necessarily $q^{*}=\frac{1+\Delta^{*}}{2}>\frac{1}{2}$. In both scenarios and conditional on non-disclosure the board rejects the project if and only if $s=0$. However, in the former case, the manager collects more information ( $q^{*}$ is higher) and hence the probability of disclosing $\omega=1$ is strictly higher. Therefore, the overall probability of project's approval is lower when the board has reputation concerns. This completes the proof.

Consider the statement on shareholders' value. According to (A5), for a given level of $q$ and $\Delta$ shareholders' value satisfies

$V(q, \Delta)=1-(1-p)\left[\frac{q}{1-q}-\lambda\left(\frac{q}{1-q} \alpha(q, 1, \Delta)-\alpha(q, 0, \Delta)\right)-\frac{2 q-1}{1-q}(1-\lambda) \cdot 1_{\left\{q \geq \frac{1+\Delta}{2}\right\}}\right]$

where $\alpha(q, p, \Delta)$ is given by $(\mathrm{A} 13)$. Note that $V(0, \Delta)=1$ for any $\Delta$ and that $V\left(\frac{1}{1+\frac{\pi}{1-\pi} \frac{1-\Delta}{1+\Delta}}, \Delta\right)$ and $V\left(\frac{1+\Delta}{2}, \Delta\right)$ decrease in $\Delta$. The arguments in the previous paragraphs reveal that in equilibrium:

1. If $p<\frac{1}{2}$ and $\lambda<\frac{1}{2 \pi}$ then $q^{*}=0$ when the board has no concerns for reputation, and otherwise $q^{*} \in\left\{0, \frac{1}{1+\frac{\pi}{1-\pi} \frac{1-\Delta^{*}}{1+\Delta^{*}}}\right\}$ where $\Delta^{*}<0$.

2. If $p \geq \frac{1}{2}$ and $\lambda<\frac{1}{\pi(3-2 \pi)}$ then $q^{*}=\frac{1}{2}$ when the board has no concerns for reputation, and otherwise $q^{*}=\frac{1+\Delta^{*}}{2}$ where $\Delta^{*}>0$.

3. Else, $q^{*}=1-\pi$ when the board has no concerns for reputation, and otherwise $q^{*}=\frac{1}{1+\frac{\pi}{1-\pi} \frac{1-\Delta^{*}}{1+\Delta^{*}}}$ where $\Delta^{*}<0$.

It follows that in the first and the second cases shareholders' welfare decreases when the board has reputation concerns, and in the third case, it strictly increases. 
Proof of Proposition 5. The analysis with transparency is not fundamentally different from the analysis without transparency. The main difference comes form how shareholders update their beliefs given the board's action. In particular, Lemma A.4 applies with the following adjustments. First, condition (A10) becomes

$$
\Delta^{*} \equiv \Gamma\left(\frac{1}{1+\frac{1-\lambda}{\lambda} \frac{1}{q^{*}(1-\pi)+\left(1-q^{*}\right) \pi}}\right)-\Gamma(1)
$$

and condition and (A12) becomes

$$
\Delta^{*}=\Gamma(1)-\Gamma\left(\frac{1}{1+\frac{1-\lambda}{\lambda} \frac{1}{q^{*} \pi+\left(1-q^{*}\right)(1-\pi)}}\right)
$$

In both cases, $\Delta^{*}$ is bigger in absolute terms compared to the original and corresponding terms in Lemma A.4. ${ }^{25}$ In addition, unlike Lemma A.9, off-equilibrium events could potentially matter, since conditional on non-disclosure the board might, for example, reject the project with probability one. Nevertheless, as long as $0 \leq \Gamma(0)<\Gamma(1)<1$ then it is guaranteed that $\Delta^{*}<1$ and, as I argue next, the set of equilibria is generically the same. Indeed, consider an equilibrium in which $q=0$ and note that even the most optimistic board, an expert board with signal $s=1$, will reject the project as long as $0<\frac{1}{1+\frac{\pi}{1-\pi} \frac{1-\Delta^{*}}{1+\Delta^{*}}} \Leftrightarrow \Delta^{*}<\frac{1}{2 \pi-1}$ which always holds when $\pi \in\left(\frac{1}{2}, 1\right)$ and $\Delta^{*}<1$. Thus, regardless of the off-equilibrium beliefs, this equilibrium exists under the same conditions as in Lemma A.4 part (i). All other equilibria do not involve offequilibrium beliefs and hence the only difference comes from conditions (A20) and (A21). Note that since for a given set of parameters $\left|\Delta^{*}\right|$ is higher with transparency, conditions (A8), (A9), and (A11) are less likely to be satisfied. So sometimes an equilibrium without transparency could exist where an equilibrium with transparency does not. Nevertheless, Lemma 5 can be easily modified to account for transparency. In what follows I restrict attention to parameters

\footnotetext{
${ }^{25}$ Note that condition (A12) does not guarantee a unique solution but condition (A21) does.
} 
such that equilibrium with and without transparency exists.

Consider the proof of Proposition 4. It shows that in equilibrium $\Delta^{*}>0$ if and only if $p \geq \frac{1}{2}$ and $\lambda<\frac{1}{\pi(3-2 \pi)}$. Since $q^{*}$ increases with $\Delta^{*}$ and since transparency increases $\left|\Delta^{*}\right|$ then transparency reduces the amount of information that is collected by the manager in equilibrium (that is, a higher $q^{*}$ ) if and only if $p \geq \frac{1}{2}$ and $\lambda<\frac{1}{\pi(3-2 \pi)}$. Noting that the ex-ante likelihood of disclosure is ep, completes the first part of the proposition. The proof of Proposition 4 also shows that $V\left(q^{*}\left(\Delta^{*}\right), \Delta^{*}\right)$ decrease in $\Delta^{*}$ and that reputation concerns harm shareholders' value if and only if $\lambda<\lambda^{\dagger}$ where $\lambda^{\dagger}$ is given by (5). Thus, if $p<\frac{1}{2}$ and $\lambda \geq \frac{1}{2 \pi}$, or $p \geq \frac{1}{2}$ and $\lambda \geq \frac{1}{\pi(3-2 \pi)}$, then shareholders' value increases when the board has reputation and $\Delta^{*}<0$. Therefore, lower $\Delta^{*}$ amplifies the positive effect of reputation. When $p \geq \frac{1}{2}$ and $\lambda<\frac{1}{\pi(3-2 \pi)}$ then shareholders' value decreases when the board has reputation and $\Delta^{*}>0$. Therefore, higher $\Delta^{*}$ amplifies the negative effect of reputation. Only when $p<\frac{1}{2}$ and $\lambda<\frac{1}{2 \pi}$ then shareholders' value decreases when the board has reputation and $\Delta^{*}<0$. In that case, transparency mitigates the negative effect of reputation. This establishes the result. 


\section{Supplemental Results}

Lemma A.5 Let $h(\cdot \mid \theta)$ be any strictly positive and continuous probability density function over $\left[\frac{1}{2}, 1\right]$ where $\theta \in \Theta$ is a compact set of parameters. Then, for any $\omega \in\{0,1\}$ and $s \in[0,1]$

$$
f_{\omega}(s \mid \theta) \equiv\left[h(1-s \mid \theta) \cdot 1_{\left\{s \leq \frac{1}{2}\right\}}+h(s \mid \theta) \cdot 1_{\left\{s>\frac{1}{2}\right\}}\right][\omega s+(1-\omega)(1-s)]
$$

is a valid probability density function and it satisfies the MLRP. Moreover, there exists a nonzero measure set of parameters $\Theta^{\prime} \subset \Theta$ such that for any $\theta \in \Theta^{\prime}$ there are $0 \leq p_{1}(\theta)<p_{2}(\theta) \leq$ $\frac{1}{2}$ and $p_{3}(\theta) \in\left(\frac{1}{2}, 1\right]$ such that if $p \in\left(p_{1}(\theta), p_{2}(\theta)\right) \cup\left(p_{3}(\theta), 1\right)$ then $\alpha(p, p \mid \theta)>p$.

Proof. I omit $\theta$ to ease the exposition. First note that $f_{\omega}(s)$ is a valid density function. Indeed, given the properties of $h(\cdot)$ then $f_{\omega}(s)$ is also strictly positive and continuous function, and

$$
\begin{aligned}
\int_{0}^{1} f_{\omega}(s) d s & =\int_{0}^{\frac{1}{2}} h(1-s)[\omega s+(1-\omega)(1-s)] d s+\int_{\frac{1}{2}}^{1} h(s)[\omega s+(1-\omega)(1-s)] d s \\
& =\int_{\frac{1}{2}}^{1} h(s)[\omega(1-s)+(1-\omega) s] d s+\int_{\frac{1}{2}}^{1} h(s)[\omega s+(1-\omega)(1-s)] d s \\
& =\int_{\frac{1}{2}}^{1} h(s) d s=1
\end{aligned}
$$

Second, note that the likelihood function is given by $l(s)=\frac{s}{1-s}$ and hence $s^{*}(p)=1-p$. For any $p \in[0,1]$ I have

$$
\alpha(p, p)=\int_{1-p}^{1}\left[p f_{1}(s)+(1-p) f_{0}(s)\right] d s
$$

Let $\Theta=\left[0, \frac{1}{4}\right]^{2}$ and $\theta=(\Delta, \varepsilon)$ and suppose $\int_{\frac{1}{2}+\Delta}^{1-\Delta} h(s) d s=1-\varepsilon$. Consider $p \in\left(\frac{1}{2}-\Delta, \frac{1}{2}\right)$ 
then

$$
\begin{aligned}
\alpha(p, p) & =\int_{1-p}^{1} h(s)[s p+(1-s)(1-p)] d s>\int_{\frac{1}{2}+\Delta}^{1-\Delta} h(s)[s p+(1-s)(1-p)] d s \\
& >\int_{\frac{1}{2}+\Delta}^{1-\Delta} h(s)[(1-\Delta) p+\Delta(1-p)] d s=[(1-\Delta) p+\Delta(1-p)](1-\varepsilon)
\end{aligned}
$$

Thus, $\alpha(p, p)>p \Leftrightarrow p<\frac{\Delta(1-\varepsilon)}{1-(1-2 \Delta)(1-\varepsilon)}$ where $\frac{\Delta(1-\varepsilon)}{1-(1-2 \Delta)(1-\varepsilon)} \in\left(0, \frac{1}{2}\right)$. Thus, if $\varepsilon<\min \left\{\frac{4 \Delta^{2}}{(1-2 \Delta)^{2}+2 \Delta}, \frac{1}{4}\right\}$ then the interval $\left[\frac{1}{2}-\Delta, \frac{\Delta(1-\varepsilon)}{1-(1-2 \Delta)(1-\varepsilon)}\right]$ is not empty and $\left(p_{1}, p_{2}\right)$ as in the statement do exist. Consider $p \in[1-\Delta, 1]$ then

$$
\begin{aligned}
\alpha(p, p) & =\int_{1-p}^{\frac{1}{2}} h(1-s)[p s+(1-p)(1-s)] d s+\int_{\frac{1}{2}}^{1} h(s)[p s+(1-p)(1-s)] d s \\
& =\int_{p}^{1} h(s)[p s+(1-p)(1-s)] d s+\int_{\frac{1}{2}}^{p} h(s) d s>\left[p^{2}+(1-p)^{2}\right] \int_{p}^{1} h(s) d s+\int_{\frac{1}{2}}^{p} h(s) d s \\
& =\int_{p}^{1} h(s) d s-2 p(1-p) \int_{p}^{1} h(s) d s+\int_{\frac{1}{2}}^{p} h(s) d s=1-2 p(1-p) \int_{p}^{1} h(s) d s
\end{aligned}
$$

Note that $1-2 p(1-p) \int_{p}^{1} h(\pi) d \pi>p \Leftrightarrow \frac{1}{2 p}>\int_{p}^{1} h(\pi) d \pi$. But if $p>1-\Delta$ then $\int_{p}^{1} h(\pi) d \pi<$ $\varepsilon$ and therefore, $\int_{p}^{1} h(\pi) d \pi<\varepsilon<\frac{1}{2} \leq \frac{1}{2 p}$. Thus $p_{3}$ as required exists as well.

Lemma A.6 If $p \neq \beta$ then $e^{*}$ increases in $p$.

Proof. Suppose $p \neq \beta$ and consider a small increase in $p$. Unless the constraint $q \leq p$ binds, (A2) implies that $q_{\lambda}^{*}$ is invariant to $p$. Therefore, $e^{*}$ must increase in order to keep $q$ on the same level of $q^{*}$. If $q^{*}=p$ then $e^{*}=0$ and it can only increase.

\section{Lemma A.7 (Supplement to Proposition 2)}

(i) $V\left(\lambda, e^{*}\right)$ increases with $\bar{e}$.

(ii) If $p \geq \frac{1}{2}, \bar{e}=1$, and $1<\max _{q \in\left(0, \frac{1}{2}\right)}\left\{\frac{\alpha(q, q)}{q}\right\}<\min _{q \in\left[\frac{1}{2}, p\right]}\left\{\frac{1-\alpha(q, q)}{1-q}\right\}$ then $\lambda^{*}<1$. 
Proof. Consider part (i). If $\bar{e}$ is higher then the manager can also choose from $[\underline{q}-\varepsilon, q]$. By revealed preferences, and since the choice of $q_{\lambda}^{*}$ is still available, the manager will potentially switch to $q \in[\underline{q}-\varepsilon, \underline{q}]$. Therefore, $q_{\lambda}^{*}$ is decreases in $\bar{e}$. According to Lemma 3, this implies that $V\left(\lambda, q_{\lambda}^{*}\right)$ increases with $\bar{e}$.

Consider part (ii). First, if $1<\max _{q \in\left(0, \frac{1}{2}\right)}\left\{\frac{\alpha(q, q)}{q}\right\}$ then there exists $q^{\prime} \in\left(0, \frac{1}{2}\right)$ such that $\frac{1-\alpha\left(q^{\prime}, q^{\prime}\right)}{1-q^{\prime}}<1$. This implies $q_{1}^{*}=q_{\min }>0$. Second, since $1<\min _{q \in\left[\frac{1}{2}, p\right]}\left\{\frac{1-\alpha(q, q)}{1-q}\right\}$ then $\frac{1-\alpha\left(q^{\prime}, q^{\prime}\right)}{1-q^{\prime}}<\min _{q \in\left[\frac{1}{2}, p\right]}\left\{\frac{1-\alpha(q, q)}{1-q}\right\}$. Hence, if $\lambda=1$ the manager strictly prefers $q^{\prime}>0$ over any $q \in\left[\frac{1}{2}, p\right]$. Thus, $q_{\min }<\frac{1}{2}$ and $\lambda^{+}<1$. Moreover, $q_{1}^{*}>0 \Rightarrow V\left(q_{1}^{*}, 1\right)<1$. Third, according to $(\mathrm{A} 4), \lambda^{+}$satisfies $\frac{\frac{1}{\lambda^{+}}-\alpha\left(q_{\lambda^{+}}^{*}, q_{\lambda^{+}}^{*}\right)}{1-q_{\lambda^{+}}^{*}}=\min _{q \in\left[\frac{1}{2}, p\right]}\left\{\frac{1-\alpha(q, q)}{1-q}\right\}$ where $q_{\lambda^{+}}^{*} \in \arg \min _{q \in\left[0, \frac{1}{2}\right)} \frac{\frac{1}{\lambda^{+}}-\alpha(q, q)}{1-q}$. If on the contrary $q_{\lambda^{+}}^{*}>0$ then $\frac{1}{\lambda^{+}}>\frac{\frac{1}{\lambda^{+}}-\alpha\left(q_{\lambda^{+}}^{*}, q_{\lambda^{+}}^{*}\right)}{1-q_{\lambda^{+}}^{*}}$ and hence $\frac{\alpha\left(q_{\lambda^{+}}^{*} q_{\lambda^{+}}^{*}\right)}{q_{\lambda^{+}}^{*}}>\frac{1}{\lambda^{+}}$. Overall, $\frac{\alpha\left(q_{\lambda^{+}}^{*} q_{\lambda^{+}}^{*}\right)}{q_{\lambda^{+}}^{*}}=\min _{q \in\left[\frac{1}{2}, p\right]}\left\{\frac{1-\alpha(q, q)}{1-q}\right\}$ which contradicts $\max _{q \in\left(0, \frac{1}{2}\right)}\left\{\frac{\alpha(q, q)}{q}\right\}<\min _{q \in\left[\frac{1}{2}, p\right]}\left\{\frac{1-\alpha(q, q)}{1-q}\right\}$. I conclude that $q_{\lambda^{+}}^{*}=0$ and hence $V\left(q_{\lambda^{+}}^{*}, \lambda^{+}\right)=1>V\left(q_{1}^{*}, 1\right)$ implying that $\lambda^{*}<1$.

\section{Lemma A.8 (Supplement to Proposition 3)}

(i) $\bar{\lambda} \leq 1$ if and only if there exists $q \in\left(0, \min \left\{p, \frac{1}{2}\right\}\right]$ such that $\alpha\left(q, q, \frac{1}{2}\right) \geq q$. If $\lim _{q \rightarrow 0} \frac{\partial \alpha\left(q, q, \frac{1}{2}\right)}{\partial q}=\infty$ then $\bar{\lambda}=0$.

(ii) Suppose $p>\frac{1}{2}$ and $\lim _{q \rightarrow 0} \frac{\partial \alpha\left(q, q, \beta_{0}\right)}{\partial q}<\infty$ for $\beta_{0} \equiv \frac{p+1}{2} \cdot{ }^{26}$ Then, there exist $\lambda_{0} \in\left(0, \lambda^{+}\right)$ and $e_{\lambda_{0}}<1$ such that $\beta^{*} \in\left(\frac{1}{2}, \beta_{0}\right]$ for all for all $\bar{e} \in\left(e_{\lambda_{0}}, 1\right)$.

Proof. Consider part (i). Note that by its definition $\bar{\lambda} \leq 1$ if and only if there exists $q \in\left(0, \min \left\{p, \frac{1}{2}\right\}\right]$ such that $\alpha\left(q, q, \frac{1}{2}\right) \geq q$. Also, since $\frac{q}{\alpha\left(q, q, \frac{1}{2}\right)}>0$ for all $q>0$, then

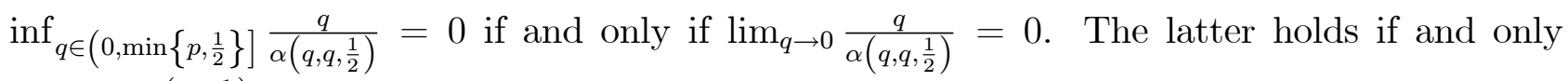
$\lim _{q \rightarrow 0} \frac{\partial \alpha\left(q, q, \frac{1}{2}\right)}{\partial q}=\infty$.

Consider part (ii) and let $\lambda_{0} \in\left(0, \min \left\{\left(1+\max _{q \in[0, p]} \frac{\partial \alpha\left(q, q, \beta_{0}\right)}{\partial q}\right)^{-1}, \lambda^{+}\right\}\right)$. Since $\frac{\partial \alpha\left(q, q, \beta_{0}\right)}{\partial q}>$ 0, $F_{\omega}$ is continuous, and $\lim _{q \rightarrow 0} \frac{\partial \alpha\left(q, q, \beta_{0}\right)}{\partial q}<\infty$ then $\lambda_{0}$ is well defined. Recall, the man-

\footnotetext{
${ }^{26}$ Note that $\lim _{q \rightarrow 0} \frac{\partial \alpha(q, q, \beta)}{\partial q}<\infty$ if, for example, $F_{i}=F_{i}(x)=1-e^{-\mu_{i} x}$ and $\mu_{0}>\mu_{1}$.
} 
ager chooses $q \in[q(\bar{e}), p]$ that maximizes $U(q, \lambda ; \beta)$. When $\beta>p$ this is equivalent to minimizing $\frac{1-\lambda \alpha(q, q, \beta)}{1-q}$. Note that this function is differentiable and its derivative is given by $\frac{-\lambda \frac{\partial \alpha(q, q, \beta)}{\partial q}(1-q)+[1-\lambda \alpha(q, q, \beta)]}{(1-q)^{2}}$. If $\lambda=\lambda_{0}$ and $\beta=\beta_{0}$ then the assumption $\lambda_{0}<\frac{1}{1+\max _{q \in[0, p]} \frac{\partial \alpha\left(q, q, \beta_{0}\right)}{\partial q}}$ implies that this derivative is strictly positive on $[q(\bar{e}), p]$. Thus, the manager chooses $q(\bar{e})$, the highest learning intensity. According to Proposition 3 Part (ii), there is $e_{\lambda_{0}}<1$ such that $\beta^{*}>\frac{1}{2}$ for all $\bar{e} \in\left(e_{\lambda_{0}}, 1\right)$. Suppose $\bar{e} \in\left(e_{\lambda_{0}}, 1\right)$ and note that based on the argument above the manager chooses $q(\bar{e})$ when $\lambda=\lambda_{0}$ and $\beta=\beta_{0}$. Since $\beta_{0}>\frac{1}{2}$ then any $\beta>\beta_{0}$ yields a strictly lower value for shareholder (more over-rejection). Therefore, $\beta^{*} \in\left(\frac{1}{2}, \beta_{0}\right]$ as required.

Lemma A.9 (Equilibrium Selection) In any equilibrium (subject to the restrictions of Section III in the main text) the probability that the project is approved is strictly between zero and one.

Proof. Recall that $\operatorname{Pr}[a=1]$ is the manager's expected utility. The manager can always choose $e=\bar{e}$, learn with probability $\bar{e} p$ that $\omega=1$, disclose this information to the board, and thereby guarantee the approval of the project. Thus, in any equilibrium $\operatorname{Pr}[a=1] \geq \bar{e} p>0$. Next, suppose on the contrary that $\operatorname{Pr}[a=1]=1$. The proof proceeds in several steps.

First, I argue that the probability of non-disclosure is strictly positive. Otherwise, given Lemma 1, it must be that $e=1$ and the manager discloses information about $\omega$ regardless of its realization. If so, there is a strictly positive probability that $\omega=0$ and therefore, a strictly positive probability that the board rejects the project, a contradiction.

Second, let $T(x, \lambda) \equiv \frac{1-\Gamma(x)+\Gamma(\lambda)}{1+\Gamma(x)-\Gamma(\lambda)}$ and note that conditional on non-disclosure the board with signal $s=0$ has the highest incentives to reject the project. Since the board with signal $s=0$ approves the project, it is necessary that

$$
T(\hat{\lambda}, \lambda) \geq \frac{1-q}{q} \frac{\pi}{1-\pi}
$$


where $\hat{\lambda} \equiv \operatorname{Pr}[\rho=1 \mid a=0]$ is off-equilibrium beliefs conditional on $a=0$. Given the assumption $p<\pi$ and the observation $q \leq p$, it follows that $\frac{1-q}{q} \frac{\pi}{1-\pi}>1$. Thus, (A6) implies $T(\hat{\lambda}, \lambda)>1$ which holds if and only if $\hat{\lambda}<\lambda$. Note that conditional on observing $a=0$ the worst beliefs shareholders can have on $\rho$ are formed when the board approves the project if and only if $s=1$. These beliefs are given by $\underline{\lambda} \equiv \frac{1}{1+\frac{1-\lambda}{\lambda} \frac{1}{q(1-\pi)+(1-q) \pi}} \in(0, \lambda)$. Thus, I require $\hat{\lambda} \in[\underline{\lambda}, \lambda]$.

Third, off-equilibrium beliefs $\hat{\lambda}$ are not credible if the following condition holds: there exists $\lambda^{\prime}$ such that if conditional on the observation that $a=0$ shareholders' beliefs switch from $\hat{\lambda}$ to $\lambda^{\prime}$ and at the same time it is optimal for the board to behaves consistently with those beliefs given the manager's equilibrium effort. In what follows I demonstrate that any beliefs $\hat{\lambda} \in[\underline{\lambda}, \lambda]$ are not credible in the above sense. Consider alterative beliefs in which shareholders expect that the expert board approves the project if and only if $s=1$ and the layman board approves the project with probability $\delta$. Then, $\operatorname{Pr}[\rho=1 \mid a=0]$ is given by

$$
\lambda^{\prime}(\delta) \equiv \frac{1}{1+\frac{1-\lambda}{\lambda} \frac{1-\delta}{q(1-\pi)+(1-q) \pi}}
$$

Note that $\lambda^{\prime}(\delta)$ spans $[\underline{\lambda}, 1]$ and it increases in $\delta$. Suppose on the contrary that there are credible beliefs $\hat{\lambda} \in[\underline{\lambda}, \lambda]$. If $\delta=0$ then the credibility of $\hat{\lambda}$ implies that either the board with signal $s=1$ has strict incentives to reject the project or the layman board has strict incentives to approve it. The former case holds if and only if $T(\underline{\lambda}, \lambda)<\frac{1-q}{q} \frac{1-\pi}{\pi}$ and the latter case holds if and only if $T(\underline{\lambda}, \lambda)>\frac{1-q}{q}$. Since $T(\hat{\lambda}, \lambda) \geq \frac{1-q}{q} \frac{\pi}{1-\pi}($ condition $(\mathrm{A} 6)), \hat{\lambda} \geq \underline{\lambda}$, and $T(x, \lambda)$ is decreasing in $x$, then $T(\underline{\lambda}, \lambda) \geq \frac{1-q}{q} \frac{\pi}{1-\pi}$. If $\delta=1$ then the credibility of $\hat{\lambda}$ implies that either the board with signal $s=0$ has strict incentives to approve the project or the layman board has strict incentives to reject it. The former case holds if and only if $T(1, \lambda)>\frac{1-q}{q} \frac{\pi}{1-\pi}$ and the latter case holds if and only if $T(1, \lambda)<\frac{1-q}{q}$. Thus, it has to be that $T(1, \lambda) \notin\left[\frac{1-q}{q}, \frac{1-q}{q} \frac{\pi}{1-\pi}\right]$. If $\delta \in(0,1)$ then the credibility of $\hat{\lambda}$ implies that it has to be that $T\left(\lambda^{\prime}(\delta), \lambda\right) \neq \frac{1-q}{q}$. That is, the layman board is not indifferent. Since $T(x, \lambda)$ is decreasing in $x$ and $\lambda^{\prime}(\delta)$ spans 
$[\underline{\lambda}, 1]$ then either $T(\underline{\lambda}, \lambda)<\frac{1-q}{q}$ or $T(1, \lambda)>\frac{1-q}{q}$. Since $T(\underline{\lambda}, \lambda) \geq \frac{1-q}{q} \frac{\pi}{1-\pi}>\frac{1-q}{q}$ it must be that $T(1, \lambda)>\frac{1-q}{q}$. Since $T(1, \lambda) \notin\left[\frac{1-q}{q}, \frac{1-q}{q} \frac{\pi}{1-\pi}\right]$, I conclude that $\frac{1-q}{q} \frac{\pi}{1-\pi}<T(1, \lambda)$. Since $\frac{1-q}{q} \frac{\pi}{1-\pi}>1$ then $T(1, \lambda)>1 \Leftrightarrow \Gamma(\lambda)>\Gamma(1)$ which never holds, a contradiction.

Lemma A.10 ("Small" Direct Effect) Consider the model in Section III. If $p<\frac{1}{2}$ or $\lambda \leq \frac{1}{\pi} \frac{1+(2 \pi-1)^{2}}{(3-2 \pi)+(2 \pi-1)^{2}}$ then in any equilibrium the direct effect of board's reputation concerns on shareholders' welfare is zero. In all other circumstances, the direct effect is strictly negative only if $\Gamma(1)$ is sufficiently large.

Proof. The direct effect of board's reputation concerns measures the change in shareholders' value while keeping the manager's effort in collecting information on its equilibrium level in the absence of reputation concerns. Consider the three cases that are mentioned in the proof of Proposition 4. In case $1, q^{*}=0$ in the absence of reputation concerns. According to (A19) $V(0, \Delta)$ is invariant to $\Delta$ and hence reputation has no direct effect on shareholders' welfare. In case $2, q^{*}=\frac{1}{2}$ in the absence of reputation concerns, and $q^{*}=\frac{1+\Delta^{*}}{2}>\frac{1}{2}$ with reputation concerns. Moreover, according to Lemma A.4 part (iii), condition (A11) must hold. Therefore, $0<\Delta^{*} \leq 2 p-1$. Since $p<\pi$ then $<2 \pi-1$. Note that $\frac{1}{2} \in\left[\frac{1}{1+\frac{\pi}{1-\pi} \frac{1-\Delta^{*}}{1+\Delta^{*}}}, \frac{1}{1+\frac{1-\pi}{\pi} \frac{1-\Delta^{*}}{1+\Delta^{*}}}\right)$ if and only if $\left|\Delta^{*}\right|<2 \pi-1$ which holds. Therefore, according to (A19) and (A13) $V\left(\frac{1}{2}, \Delta^{*}\right)=V\left(\frac{1}{2}, 0\right)$ and there is no direct effect.

In case $3, q^{*}=1-\pi$ in the absence of reputation concerns, and $q^{*}=\frac{1}{1+\frac{\pi}{1-\pi} \frac{1-\Delta^{*}}{1+\Delta^{*}}}<1-\pi$ with reputation concerns. Lemma A.4 part (ii) implies that either (A8) or (A9) holds. This implies that $1-\pi \in\left[\frac{1}{1+\frac{\pi}{1-\pi} \frac{1-\Delta^{*}}{1+\Delta^{*}}}, \frac{1}{1+\frac{1-\pi}{\pi} \frac{1-\Delta^{*}}{1+\Delta^{*}}}\right)$. Thus, according to (A19) and (A13) I have

$$
V\left(1-\pi, \Delta^{*}\right)=V(1-\pi, 0)-\frac{2(1-\pi)-1}{\pi}(1-\lambda) \cdot 1_{\left\{\Delta^{*} \leq 1-2 \pi\right\}}
$$

If $\Delta^{*}>1-2 \pi$ then reputation has no direct effect on shareholders' value, and otherwise, the 
direct effect is negative. Note that if $p<\frac{1}{2}$ and $\frac{1}{\pi(3-2 \pi)}>\lambda>\frac{1}{2 \pi}$ then condition (A9) is satisfied but not condition (A8). Since $1-2 \pi<2 p-1$ if and only if $1-\pi<p$ then in this case $1-\pi<\frac{1+\Delta^{*}}{2}$ and there is no direct effect. If $\lambda \geq \frac{1}{\pi(3-2 \pi)}$ then the combination of conditions (A8) and (A9) becomes

$$
\max \left\{\frac{1-\lambda \pi(3-2 \pi)}{(1-\lambda \pi)(2 \pi-1)}, \frac{p-\pi}{p(1-\pi)+\pi(1-p)}\right\}<\Delta^{*}<0
$$

Note that $\frac{p-\pi}{p(1-\pi)+\pi(1-p)}>1-2 \pi \Leftrightarrow p<\frac{1}{2}$. Hence, if $p<\frac{1}{2}$ then $\Delta^{*}>1-2 \pi$ and there is no direct effect. Note that $\frac{1-\lambda \pi(3-2 \pi)}{(1-\lambda \pi)(2 \pi-1)}<1-2 \pi \Leftrightarrow \lambda>\frac{1+(2 \pi-1)^{2}}{\pi(3-2 \pi)+\pi(2 \pi-1)^{2}}$ and the latter term is greater than $\frac{1}{\pi(3-2 \pi)}$. Thus, if $p \geq \frac{1}{2}$ and $\lambda>\frac{1+(2 \pi-1)^{2}}{\pi(3-2 \pi)+\pi(2 \pi-1)^{2}}$ there are reputation functions for which $\Delta^{*}<1-2 \pi$ and (A22) is satisfied. For these functions, the direct effect is negative.

Lemma A.11 Consider the model in Section III.

(i) If $p<\frac{1}{2}, \lambda \geq \frac{1}{2 \pi}$, and $\mathcal{F}=\{a, v\}$ then there exists $\Gamma$ such that in equilibrium the board's decision making is distorted.

(ii) If $\mathcal{F}=\{v\}$ then there is no equilibrium in which the board's decision making is distorted.

Proof. Suppose $\mathcal{F}=\{a, v\}$ and consider an equilibrium in which conditional on non-disclosure the board approves the project if and only if it is expert and observes $s=1$. Let $L(a, v) \equiv$ $\operatorname{Pr}[\rho=1 \mid a, v]$. It can be verified that $L(1,1)=\frac{1}{1+\frac{1-\lambda}{\lambda} \frac{e}{e+(1-e) \pi}}, L(1,0)=1, L(0,1)=\frac{1}{1+\frac{1-\lambda}{\lambda} \frac{1}{1-\pi}}$, and $L(0,0)=\frac{1}{1+\frac{1-\lambda}{\lambda} \frac{1}{\pi}}$. For example, if $(a, v)=(1,0)$ then shareholders infer that $\omega=0$ and that information was not disclosed. Therefore, it must be the board is an expert. Also, note that $L$ increases in $a$ and decreases in $v$. Let $q(e, s)$ be the board's beliefs conditional on non-disclosure and conditional on its private information, if exists. Note that

$$
\mathbb{E}\left[u_{B}(a, \omega) \mid s\right]= \begin{cases}q(e, s)[1+\Gamma(L(1,1))]+(1-q(e, s)) \Gamma(L(1,0)) & \text { if } a=1 \\ q(e, s) \Gamma(L(0,1))+(1-q(e, s))[1+\Gamma(L(0,0))] & \text { if } a=0\end{cases}
$$


Thus, in this proposed equilibrium and given $e$, the board approves the project if and only if $q(e, s) \geq \frac{1+\Delta\left(e^{*}\right)}{2}$ where $e^{*}$ is the equilibrium level of effort and

$$
\Delta\left(e^{*}\right)=\frac{\Gamma(L(0,0))-\Gamma(L(1,0))-\Gamma(L(1,1))+\Gamma(L(0,1))}{2+\Gamma(L(0,0))-\Gamma(L(1,0))+\Gamma(L(1,1))-\Gamma(L(0,1))}
$$

and note that only $L(1,1)$ is a function of $e^{*}$ and that $\Delta\left(e^{*}\right)<0$. So, the board is biased toward approval of the project. In equilibrium $\Delta\left(e^{*}\right)$ is fixed from the manager's and the board's point of view. Lemma A.4 part (ii) can be applied with the exception that instead of condition (A10), condition (A23) must hold where $e^{*}$ satisfies $q\left(e^{*}\right)=\frac{1}{1+\frac{\pi}{1-\pi} \frac{1-\Delta}{1+\Delta}}$. Note that $q\left(e^{*}\right)$ increases with $\Delta$ and hence $e^{*}$ decreases with $\Delta$. Since $L(1,1)$ decreases in $e$ and $\Gamma$ is an increasing function, then the r.h.s of (A23) is decreasing in $\Delta$. Therefore, a solution exists for the above fixed problem. Last, as in Lemma A.4, conditions (A8) and (A9) must hold. Since $p<\frac{1}{2}$ and $\lambda \geq \frac{1}{2 \pi}$ then condition (A9) holds. Moreover, note that if $\Gamma(1)>0$ is sufficiently small, then so is the absolute value of $\left|\Delta\left(e^{*}\right)\right|$. Therefore, there exists $\Gamma$ such that the proposed equilibrium exists. This proves first part.

Suppose $\mathcal{F}=\{v\}$ and note that

$\mathbb{E}\left[u_{B}(a, \omega) \mid s\right]= \begin{cases}q(e, s)[1+\Gamma(\operatorname{Pr}[\rho=1 \mid v=1])]+(1-q(e, s)) \Gamma(\operatorname{Pr}[\rho=1 \mid v=0]) & \text { if } a=1 \\ q(e, s) \Gamma(\operatorname{Pr}[\rho=1 \mid v=0])+(1-q(e, s))[1+\Gamma(\operatorname{Pr}[\rho=1 \mid v=1])] & \text { if } a=0\end{cases}$

thus, as long as $0 \leq \Gamma(0)<\Gamma(1) \leq 1$ and regardless of $\Gamma(\operatorname{Pr}[\rho=1 \mid v=1])$ and $\Gamma(\operatorname{Pr}[\rho=1 \mid v=0])$, the board will approve the project if and only if $q(e, s) \geq \frac{1}{2}$. This means that the board is maximizing the probability that $a=\omega$. Since the expert board has more information, it follows that an equilibrium always exists, and in this equilibrium $\operatorname{Pr}[\rho=1 \mid v=1]>\operatorname{Pr}[\rho=1 \mid v=0]$. This proves the second part.

Lemma A.12 Consider the model in Section III and suppose the manager's power is rede- 
fined as $\operatorname{Pr}[a=1 \mid \omega=0]$. There are circumstances in which manager's power increases on the expense of shareholders' value.

Proof. For a given $q$ and $\Delta$,

$\operatorname{Pr}[a=1 \mid \omega=0]=\lambda\left[\left(p-\frac{p-q}{1-q}\right) \alpha(q, 1, \Delta)+\left[1-p+\frac{p-q}{1-q}\right] \alpha(q, 0, \Delta)\right]+(1-\lambda) \cdot 1_{\left\{q \geq \frac{1}{2}\right\}}$

If $p \geq \frac{1}{2}$ and $\lambda<\frac{1}{\pi(3-2 \pi)}$ then according to Proposition 4 reputation harms shareholders' value. According to the proof of Proposition 4, without reputation concerns the manager chooses $q^{*}=\frac{1}{2}$ and with reputation he chooses $q^{*}=\frac{1+\Delta^{*}}{2}$ where $\Delta^{*}>0$. Using (A13) one can verify that $\operatorname{Pr}[a=1 \mid \omega=0]$ is strictly smaller in the former case. Thus, even under the alternative definition, manager's power can increase on the expense of shareholders' value. 